\title{
Die Handschriftenakquisitionen des Septuaginta-Unternehmens am Beispiel der Orientreise Martin Flashars im Jahr 1914*
}

\author{
Felix Albrecht
}

Das Bildarchiv des Septuaginta-Unternehmens der Akademie der Wissenschaften zu Göttingen ist in seiner Art weltweit einzigartig. Neben zahlreichen Mikroverfilmungen von Septuagintahandschriften besitzt das Unternehmen eine beeindruckende Anzahl an alten Handschriftenphotographien, die noch aus der Gründerzeit des Unternehmens stammen. Der besondere Wert dieser Aufnahmen besteht insbesondere darin, dass ein Teil der photographierten Handschriften inzwischen unzugänglich oder gar verschollen ist. Dies betrifft vornehmlich Handschriften aus Bibliotheken des östlichen Mittelmeerraums. Die Beschaffung des handschriftlichen Materials gehört zu den grundlegenden Aufgaben des Septuaginta-Unternehmens. Die Wege sind damals wie heute dieselben: Zunächst wird versucht, über die entsprechenden Bibliotheken an Photographien der Handschriften zu gelangen. Scheitern diese mitunter langwierigen Versuche, müssen die Bibliotheken vor Ort aufgesucht werden. So werden beispielsweise gegenwärtig Expeditionen in die Mönchsrepublik Athos unternommen, um die Bestände des Unternehmens zu komplementieren und neue Handschriften zu akquirieren.

\section{Die Handschriftenreisen vor dem 1. Weltkrieg}

Bereits 1909 bemerkte Alfred Rahlfs, dass „Nachforschungen an Ort und Stelle“ notwendig seien, um die noch nicht oder zumindest unzureichend erschlossenen Handschriftenbestände zu erforschen: „Da die gedruckten Kataloge oft nicht genügen und von verschiedenen Handschriftenbeständen überhaupt noch keine Kataloge erschienen sind, so weist unser Inventar naturgemäß noch manche Lücke auf, die erst mit der Zeit durch Nachforschungen an Ort und Stelle ausgefuillt werden können“ ${ }^{1}{ }^{1}$ Um diese ,Lücken' zu schließen, wurden Reisen nach Paris ${ }^{2}$,

* Der Abdruck der Abbildungen 1-4 erfolgt mit freundlicher Genehmigung des Septuaginta-Unternehmens der Akademie der Wissenschaften zu Göttingen.

1 Kommission des Septuaginta-Unternehmens, Bericht über das Septuaginta-Unternehmen der Kgl. Gesellschaft der Wissenschaften zu Göttingen, in: NGWG. Geschäftliche Mitteilungen 1909, Berlin 1909, 129-138, hier 134.

2 Zu Walter Reimpells Reise nach Paris vgl. Kommission des Septuaginta-Unternehmens, Dritter Bericht über das Septuaginta-Unternehmen (Berichtsjahr 1910), in: 
in den Orient ${ }^{3}$ und auf den Athos ${ }^{4}$ unternommen, so dass Rahlfs bereits drei Jahre später vermelden konnte, das Unternehmen besitze Photographien aus circa 70 Handschriften. ${ }^{5}$ Und für 1913 vermerkt er: „Der Fonds von Photographien griechischer Handschriften hat sich bedeutend vermehrt: zu den im vorigen Berichte erwähnten 70 Handschriften sind weitere 70 hinzugekommen “. ${ }^{6} \mathrm{Im}$ Jahr 1914 schließlich fanden zwei bedeutende Expeditionen statt, die den Bestand des Unternehmens nochmals erweiterten: Eine von Martin Flashar und Hugo Duensing unternommene Reise in den Orient sowie eine Reise Carl Schmidts auf den Sinai. ${ }^{7}$ Die Orientreise Flashars und Duensings am Vorabend des 1. Weltkriegs

NGWG. Geschäftliche Mitteilungen 1911, Berlin 1911, 21-25, hier 23: „Herr Reimpell [ist] im Januar und Februar 1911 etwa 4 Wochen lang in Paris gewesen“. - Zu Walter Reimpell s.u. Anm. 9.

3 Bei den frühen Orientreisen im Dienste des Unternehmens handelt es sich um den (erfolglosen) Auftrag des Franz Nikolaus Finck, die armenischen Handschriften des Alten Testaments zu katalogisieren. Vgl. dazu Kommission des Septuaginta-Unternehmens, Dritter Bericht (Berichtsjahr 1910), 22: „Leider haben die von Herrn Prof. Finck unternommenen Nachforschungen nach armenischen Handschriften im Orient, von denen viel erhofft wurde, nur geringen Erfolg gehabt“. Im der Internationalen Assoziation der Akademien zur Generalversammlung 1913 vorgelegten Bericht heißt es: „Die Inventarisierung der armenischen Handschriften hatte Herr Prof. Franz Nikolaus Finck in Berlin übernommen. Er hatte eine Reihe von Armeniern mit der Aufsuchung und Beschreibung der im Orient befindlichen Handschriften beauftragt" (Kommission des SeptuagintaUnternehmens, Bericht über das Septuaginta-Unternehmen der Königl. Gesellschaft der Wissenschaften zu Göttingen. Der Internationalen Assoziation der Akademien vorgelegt zur Generalversammlung 1913, Göttingen 1913, 1-8, hier 6).

$4 \mathrm{Zu}$ Karl Dieterichs Reise zum Athos vgl. Kommission des Septuaginta-Unternehmens, Vierter Bericht über das Septuaginta-Unternehmen (Berichtsjahr 1911), in: NGWG. Geschäftliche Mitteilungen 1912, Berlin 1912, 18-20, hier 18: „Herr Dr. Karl Dieterich nahm eine Anzahl griechischer Handschriften in den Athosklöstern Lavra und Vatopedi auf“. Im Handschriftenverzeichnis notiert Rahlfs zum Kloster Vatopedi: „Einen gedruckten Katalog gibt es noch nicht, daher ist das folgende Verzeichnis gewiß sehr unvollständig. Nr. 511-515 [sc. Ra 764-769] sind von Karl Dieterich bei einem kurzen Aufenthalt an Ort und Stelle aufgenommen“; A. RAHLFs, Verzeichnis der griechischen Handschriften des Alten Testaments. Für das Septuaginta-Unternehmen aufgestellt (MSU 2), Berlin 1914, 8. Ähnlich bemerkt Rahlfs zur Lavra, ebd. 16: „Einen gedruckten Katalog gibt es noch nicht, daher ist das folgende Verzeichnis gewiß unvollständig. Die meisten Hss. sind von Karl Dieterich an Ort und Stelle aufgenommen“. - Zu Karl Dieterich s.u. Anm. 10.

5 Kommission des Septuaginta-Unternehmens, Fünfter Bericht über das SeptuagintaUnternehmen (Berichtsjahr 1912), in: NGWG. Geschäftliche Mitteilungen 1913, Berlin 1913, 23-24, hier 23: „Wir besitzen jetzt Photographien aus ungefähr 70 Handschriften“.

6 Kommission des Septuaginta-Unternehmens, Sechster Bericht über das SeptuagintaUnternehmen (Berichtsjahr 1913), in: NGWG. Geschäftliche Mitteilungen 1914, Berlin 1914, 22-24, hier 23.

7 Zur Sinai-Expedition der Preußischen Akademie der Wissenschaften im Frühjahr 1914 vgl. C. SснміDт, Bericht des wissenschaftlichen Beamten Prof. Karl Schmidt über seine Forschungsreise nach dem Katharinenkloster auf dem Sinai, in: SPAW 1915, Berlin 1915, 122-125; C. Sсhмidt / B. Moritz, Die Sinai-Expedition im Frühjahr 1914, in: SPAW.PH 7, Berlin 1926, 26-34. Vgl. Kommission des Septuaginta-Unternehmens, Siebenter Bericht über das Septuaginta-Unternehmen (Berichtsjahr 1914), in: NGWG. 
verdient dabei besondere Beachtung. ${ }^{8}$ Über sie sind wir durch einen bislang unveröffentlichten handschriftlichen Reisebericht Flashars genauestens unterrichtet, so dass es uns möglich ist, uns ein genaueres Bild jener Reise zu machen.

Die Handschriftenreisen vor dem 1. Weltkrieg im Überblick

\begin{tabular}{|c|c|c|}
\hline Paris & 1911 & $\begin{array}{l}\text { Walter Reimpell }{ }^{9} \\
\text { Forschungsaufenthalt }\end{array}$ \\
\hline Athos & 1911 & $\begin{array}{l}\text { Karl Dieterich }^{10} \\
\text { Handschriftenbeschreibungen Vatopedi (Ra 318-320; 762-763) und Lavra } \\
\text { (Ra 764-769; Cod. Athous Laurensis B 70, vermutlich auch Codd. B } \\
\text { 75-76; 87) }\end{array}$ \\
\hline
\end{tabular}

Geschäftliche Mitteilungen 1915, Berlin 1915, 19-21, hier 20: „Herr Prof. Carl Schmidt (Berlin) hat im Sommer 1914 bei einem Aufenthalt auf dem Sinai einige dort befindliche Septuaginta-Handschriften im Auftrage unseres Unternehmens photographiert und die Kopien trotz mancher Schwierigkeiten im Sept. 1914 glücklich nach Deutschland heimgebracht". Vgl. auch Kommission des Septuaginta-Unternehmens, Achter Bericht über das Septuaginta-Unternehmen (Berichtsjahr 1915), in: NGWG. Geschäftliche Mitteilungen 1916, Berlin 1916, 15-17, hier 16f. Schmidt selbst berichtet 1915: „Was nun die Aufträge anbetrifft, so wurden auf Veranlassung von Hrn. Prof. Rahlfs für das Septuagintaunternehmen verschiedene alttestamentliche Kodizes photographiert und so im ganzen 750 Aufnahmen gemacht [...]“; SснміDт, Bericht des wissenschaftlichen Beamten Prof. Karl Schmidt über seine Forschungsreise nach dem Katharinenkloster auf dem Sinai, 123. Die viermonatige Sinai-Expedition scheiterte auf tragische Weise: Durch den Ausbruch des 1. Weltkriegs überrascht, wurde das Expeditionsgut im Umfang von 30 Kisten in Suez zurückgelassen und anschließend von den Engländern entweder liquidiert oder vernichtet. Eine der Hauptaufgaben der Expedition bestand darin, die griechischen Handschriften des Sinaiklosters zu photographieren: Insgesamt wurden ca. 8000 Aufnahmen angefertigt; vgl. Schmidt / Moritz, Die Sinai-Expedition im Frühjahr 1914, 32. Im Nachbericht von 1926 heißt es dazu: „das gesamte photographische Material ist vernichtet“ (ebd. 33). Dies trifft auf das Gros der Aufnahmen zu. Schmidt selbst schreibt nun aber im Bericht von 1915: „Schließlich konnte ich wenigstens einen Teil der Schwarz-Weiß-Aufnahmen und meine schriftlichen Aufzeichnungen als Handgepäck mitnehmen"; SCHMIDT, Bericht des wissenschaftlichen Beamten Prof. Karl Schmidt über seine Forschungsreise nach dem Katharinenkloster auf dem Sinai, 124. Dabei handelte es sich ebengerade um die Photographien für das Septuaginta-Unternehmen, die - noch heute in Göttingen befindlich somit genau genommen den einzigen verbliebenen photographischen Ertrag dieser großen Forschungsreise darstellen. - Zu Carl Schmidt s.u. Anm. 12.

8 Der anschließende Ausbruch des 1. Weltkriegs markierte eine scharfe Zäsur, so dass der Erwerb neuer Handschriften gänzlich zum Erliegen kam; vgl. Kommission des SePtuagintaUnternehmens, Achter Bericht (Berichtsjahr 1915), 17: „Während die Beschaffung von Handschriften-Photographien infolge des Krieges aufgehört hat, ist die Handbibliothek des Septuaginta-Unternehmens im letzten Jahre bedeutend vermehrt worden“.

9 Zu Dr. Walter Reimpell (6.4.1886-11.12.1914) vgl. W. ReimpelL, Geschichte der babylonischen und assyrischen Kleidung, ed. E. MeYER, Berlin 1921, Vorwort; vgl. ferner Kommission des Septuaginta-Unternehmens, Siebenter Bericht (Berichtsjahr 1914), 21.

$10 \mathrm{Zu}$ Prof. Dr. Karl Dieterich (18.12.1869-16.7.1935) vgl. F. Dölger, Art. „Dieterich, Karl Gustav“, in: NDB 3, Berlin 1957, 671-672; J. Irmscher, Karl Dieterich und die Begründung der Neogräzistik in Leipzig, in: J. Werner (ed.), Modernes Griechenland, 
Die Handschriftenreisen vor dem 1. Weltkrieg im Überblick (Fortsetzung)

\begin{tabular}{lrl}
\hline $\begin{array}{l}\text { Orient } \\
\text { (Kairo, } \\
\text { Jerusalem) }\end{array}$ & 1914 & $\begin{array}{l}\text { Martin Flashar, Hugo Duensing } \\
\text { Kollationen (Ra 406; 929), Photographien (Ra 402-405; 407; 410-412) }{ }^{11}\end{array}$ \\
\hline Sinai & 1914 & $\begin{array}{l}\text { Carl Schmidt } \\
\text { Photographien (Ra 707; 710-714) }\end{array}$ \\
\hline
\end{tabular}

\section{Die Orientreise von 1914}

Über die Hintergründe der Orientreise berichtet Alfred Rahlfs: ${ }^{14}$ „Nachdem mir im Herbst 1913 mein langjähriger Freund Herr Lic. Dr. Hugo Duensing, Pastor in Dassensen bei Markoldendorf (Hannover), zu meiner Freude versprochen hatte, bei einer Studienreise nach Jerusalem, die er Anfang des Jahres 1914 antreten wollte, für das Septuaginta-Unternehmen die lange ersehnten Photographien der dortigen LXX-Hss. (mit Ausschluß der Psalterien) zu besorgen, kam ganz unvermutet von Herrn Lic. Dr. Martin Flashar, Pastor in Dechtow bei Betzin-Carwesee (Brandenburg) ein vom 28.10.1913 datierter Brief, in welchem er schrieb: ,Ich mache im Januar nächsten Jahres eine Orientreise, und möchte gern in erster Linie Septuagintastudien treiben, möglichst auch in Jerusalem. ... Dürfte ich Sie um einen Wink bitten, ob und welche Arbeiten und Kollationen im Rahmen des Septuaginta-Unternehmens möglich und wünschenswert sind.' Diese Bitte habe ich mit größter Freude erfüllt; bot sich doch hier durch eine besonders glückliche Fügung dank dem edlen und uneigennützigen Eifer eines mir bis dahin persönlich Unbekannten eine Gelegenheit, das, was mir durch Duensings Versprechen schon in Aussicht stand, noch zu vervollständigen. Denn außer den Hss., die man auf

modernes Zypern. Vorträge einer wissenschaftlichen Konferenz des Fachbereichs „Antike Literatur/Neogräzistik“ der Karl-Marx-Universität Leipzig, Amsterdam 1989, 244-261; weder Dölger noch Irmscher erwähnen den Athos-Aufenthalt Dieterichs.

11 Die Photographien der Hss. Ra 402-405; 407; 410-412 haben ein ursprüngliches Format von $18 \times 24 \mathrm{~cm}$ und sind jeweils mittig durchgeschnitten; die Gießener Kapseln haben eine Höhe von $21 \mathrm{~cm}$.

12 Zu Prof. Dr. Carl Schmidt (26.8.1868-17.4.1938) vgl. K.-G. Wesseling, Art. „Schmidt, Carl“, in: BBKL 9, Herzberg 1995, 443-446.

13 Die Photographien der Hss. Ra 707; 710-714 haben ein Format von 23,0 × 17,5 cm und die Gießener Kapseln eine Höhe von 21,0 cm. - Kurios ist die handschriftliche Notiz auf der Photorückseite von Ra 711, f. 236v, der zufolge Schmidt Eingriffe in die Handschrift nicht scheute: „Dieses Blatt habe ich losgelöst vom Deckel; da Buchstaben durch den Leim teils undeutlich. Ich gebe eine Abschrift des Textes auf dem folgenden Blatte“. In der Tat enthält das folgende Blatt eine Abschrift von der Hand Schmidts, deren Schriftzüge - wie wäre es beim ,Koptenschmidt' anders zu erwarten - seltsam ,koptisch' anmuten.

14 A. Rahlfs, Palimpsest-Fragmente des Sirach und Iob aus Jerusalem. Nach der Entzifferung von Martin Flashar, in: Kleine Mitteilungen aus dem Septuaginta-Unternehmen (MSU 1/7), Berlin 1915, 388-404 (= NGWG.PH 3, Berlin 1915, 404-420), hier 388f. [= 404f.]. 
photographischem Wege kopieren konnte, gab es in Jerusalem noch PalimpsestFragmente des Sirach und Iob, welchen auf diese Weise nicht beizukommen war. Beide waren allerdings schon durch Veröffentlichungen von J. Rendel Harris und Eugène Tisserant bekannt, aber beide unvollständig. So schlug ich Flashar vor, diese Palimpseste, vor allem die Sirach-Fragmente, zum Gegenstand seines Studiums zu wählen. Zugleich bat ich Duensing, der seit langem in der Kunst des Lesens von Palimpsesten geübt war, Flashar in diese Kunst, der er, wie ich mit Recht annahm, bis dahin ferne stand, an Ort und Stelle praktisch einzuführen und ihm speziell bei der Entzifferung der Sirach-Fragmente hilfreich zur Hand zu gehen. Flashar und Duensing gingen sofort auf meine Vorschläge ein und haben sich dann mit großem Eifer der Arbeit gewidmet. Sie trafen sich im Februar 1914 in Jerusalem, machten am 8. Februar gemeinsam einen Besuch bei dem griechischen Patriarchen Damianos und erhielten, unterstützt durch die liebenswürdige und energische Befürwortung des Herrn Generalkonsul Schmidt, die Erlaubnis zum Lesen und Photographieren aller gewünschten Hss. Nun las Duensing mit Flashar die ersten Zeilen des Sirach-Palimpsestes und zeigte ihm, wie man unlesbare Stellen durch vorsichtige Behandlung mit Schwefelammonium wieder lesbar machen kann. Sodann nahm Flashar die Arbeit auf und führte sie im wesentlichen selbständig weiter, indem er nur bei besonders schwierigen Stellen Duensings Hilfe in Anspruch nahm ...". An anderer Stelle berichtet Rahlfs über einen vorangehenden Aufenthalt Duensings in Kairo und kommt anschließend auf den Jerusalemaufenthalt beider Forscher zu sprechen: ${ }^{15}$ „Herr Duensing hat die griechische Patriarchats-Bibliothek in Kairo, über die wir nur sehr dürftige Nachrichten besaßen, aufgesucht und mehrere uns angehende Handschriften eingesehen, und er hat in Jerusalem, von wo wir bisher noch keine Handschriften-Photographien bekommen konnten, uns solche mit Überwindung nicht geringer Schwierigkeiten besorgt. Herr Flashar hat in Jerusalem mit Unterstützung des Herrn Duensing zwei Palimpsestblätter des Sirach, die erst sehr mangelhaft herausgegeben waren, mit dem besten Erfolge neu untersucht und auch unsere Kenntnis eines schlecht erhaltenen Iob-Palimpsestes erweitert". - Wer waren diese beiden Forscher, die dem Septuaginta-Unternehmen wertvolle Unterstützung leisteten?

Hugo Duensing (1877-1961), ein Schüler Julius Wellhausens und Schwiegersohn Emil Schürers, war zu der Zeit bereits ein namhafter Forscher, der sich durch äthiopistische Studien und Arbeiten zum Christlich-palästinisch-Aramäischen hervorgetan hatte. ${ }^{16} \mathrm{Zu}$ Letzterem zählte insbesondere die Göttinger Lizentiatsarbeit Duensings aus dem Jahr 1906, die unter anderem auch die Septuaginta zum Gegenstand hatte: ,Christlich-palästinisch-aramäische Texte und Fragmen-

15 Kommission des Septuaginta-Unternehmens, Sechster Bericht (Berichtsjahr 1913), 23f. - Die von Duensing in Kairo beschriebenen Handschriften erwähnt RaHLfs, Verzeichnis der griechischen Handschriften des Alten Testaments, 88.

16 Zu Dr. Hugo Duensing vgl. ausführlich F. Albrecht, Art. „Duensing, Hugo Berthold Heinrich“, in: BBKL 33, Nordhausen 2012, 307-318; hier sollen nur die wesentlichen Angaben zur Person Duensings Erwähnung finden. 
te nebst einer Abhandlung über den Wert der palästinischen Septuaginta“. Hier untersuchte Duensing einen Großteil der zum damaligen Zeitpunkt bekannten christlich-palästinisch-aramäischen Sprachreste, die in Form von Palimpsesten erhalten waren. Duensing galt - wie Rahlfs zu Recht erwähnt - als ausgewiesener Kenner im Umgang mit palimpsestierten Handschriften. Die Jerusalemreise unternahm Duensing im Rahmen des 12. Lehrkurses des „Deutschen Evangelischen Instituts für Altertumswissenschaft des Heiligen Landes" (DEI). Vom 2. Januar bis 31. Mai 1914 war Duensing daher, seit 1907 im Pfarrdienst stehend, zu Studienzwecken beurlaubt. In einem Brief an Gustaf Dalman, den damaligen Leiter des DEI, schreibt Duensing im November 1913 über seine Reiseplanungen und über die angedachten Arbeiten für das Septuaginta-Unternehmen: ${ }^{17}$

Dassensen, Post Markoldendorf, (Hannover) den 4. Nov. 1913

Hochgeehrter Herr Professor!

Vielleicht ist Ihnen schon bekannt geworden, daß ich im nächsten Jahr als Stipendiat nach Jerusalem gehe. Ich freue mich darauf, unter Ihrer sachkundigen Leitung Materien bearbeiten zu können, die mir schon lange am Herzen lagen. Gern würde ich das Thema bearbeiten: Palästina in der Mischna. Ich bin auf dies Thema zunächst durch den Umstand geraten, daß mein verstorbener Schwiegervater Schürer ein geographisches Register nebst einigen Materialien zur Geographie der Mischna mit dem Bemerken hinterließ: Ich müsse mal sehen, ob ich etwas daraus || machen könne. Nun scheint mir das außerpalästinische Material der Mischna zu geringfügig u. zu desparat als daß es sich lohnte, große Mühe darauf zu verwenden. Dagegen umfaßt das obige Thema nicht nur das umfangreichste, sondern auch das wichtigste Material, wichtig auch deshalb weil es ein Gradmesser ist für die Zuverlässigkeit der lokalen Erinnerungen der Mischna. Es wäre mir eine Freude, wenn ich diese Arbeit als Stipendiatenarbeit machen dürfte. - Daneben möchte ich mir die Abessinier in Jerusalem, auch ihre von Littmann beschriebenen Hss. ganz gern näher ansehen. Das dortige abess. Kloster hat eine lange Geschichte. Endlich möchte ich auf Bitte dem Göttinger LXXunternehmen, dessen < phot.> Apparat mir zur Verfügung steht, einige Dienste leisten. || Gearbeitet habe ich früher neben den abessinischen Sprachdokumenten hauptsächlich auf christlich-paläst. aram. Gebiete. Die semitischen Sprachen sind das Feld, mit dem ich bei Wellhausen in der philosophischen Fakultät promoviert habe, das Alte Testament hat mich als Theologen am meisten beschäftigt. - Zum Studium der LXXpalimpseste in Jerusalem kommt im Januar ein Pastor Lic. Dr. Flashar nach

17 H. Duensing an G. Dalman, Dassensen, den 4.11.1913. Quelle: DEI Jerusalem, Aktenzeichen III [1]. 
dort; seine Kraft reicht allein nicht aus. Ob wohl der Photograph dort sich in das Weißaufschwarzverfahren eingearbeitet hat? ${ }^{18}$

Am 4. Januar gedenke ich mit Herrn Stadtvikar Seeger und Herrn P. Weidenkaff aus Venedig zu fahren, am 28. aus Port Said, um am 29. in Jafa [sic!] zu sein. Falls noch nicht Wohnungen auf dem Muristan hergerichtet sind, würde ich vielleicht im Johanniterhospiz wohnen. Dort hat Herr P. Lic. Möller schon bestellt. - Von Herrn Superintendenten Russmann aus Ahlden soll ich Ihnen einen freundlichen Gruß bestellen.

Mit dem Ausdruck ausgezeichnetster Hochachtung

Ihr ergebenster

H. Duensing

Auch Martin Flashar (1885-1914), der Verfasser des uns vorliegenden Berichtes, hatte zur Septuaginta gearbeitet. Flashar wurde am 10.9.1885 als Sohn von Martin Eduard Richard Flashar (1848-1912) und seiner Ehefrau Anna, geb. Michaelis in Freystadt (Niederschlesien) geboren. ${ }^{19}$ Von 1892 bis 1904 besuchte er das Humboldt-Gymnasium in Berlin, das Victoria Gymnasium in Potsdam und das Fürstlich Stolbergsche Gymnasium in Wernigerode. Am 18.12.1904 bestand Flashar die Reifeprüfung und begann, an der Universität Breslau Theologie zu studieren. 1905 wechselte Flashar nach Halle, 1907 nach Berlin. Sein Studienschwerpunkt lag auf den orientalischen Sprachen. 1908 legte er an der Universität Halle eine von Franz Praetorius (1847-1927) betreute Inaugural-Dissertation zum Thema: „Das Ghain in der Septuaginta“ vor. Am 4.5.1913 erfolgte die Ordination, nach der Flashar zunächst als Hilfsprediger und vom 1.7.1913 bis zu seinem Tod als Pastor in Dechtow (Brandenburg) tätig war. Am 18.6.1913 heiratete er Elisabeth Ohly. Flashar fiel noch zu Beginn des 1. Weltkriegs Ende 1914 in Nordfrankreich. Alfred Rahlfs, der die Arbeiten Flashars an den Jerusalemer

18 Zum Weißaufschwarzverfahren vgl. K. Krumbacher, Die Photographie im Dienste der Geisteswissenschaften, in: J. Ilberg (ed.), Neue Jahrbücher für das klassische Altertum, Geschichte und deutsche Literatur 9 (NJKA 17), Leipzig 1906, 601-659 (Mit fünfzehn Tafeln), hier 621-633.

19 Zu Dr. Martin Flashar vgl. F. Albrecht, Art. „Flashar, Martin Johannes Gerhard“, in: BBKL 33, Nordhausen 2012, 418-420. - Leider war die kirchliche Personalakte Martin Flashars nicht auffindbar: Weder im Ev. Landeskirchlichen Archiv Berlin (nachrichtl. K. Köhler, 18.07.2011) noch im Geheimen Staatsarchiv Preußischer Kulturbesitz (Konsistorialakten bis 1889; nachrichtl. M. Leibetseder, 01.08.2011), resp. im Brandenburgischen Landeshauptarchiv (Konsistorialakten bis 1920, nachrichtl. W. Heegewaldt, 02.08.2011). Der Konsistorialbestand wurde im 2. Weltkrieg stark beschädigt, so dass mit Verlust der Akte Flashar zu rechnen ist. Auch im Archiv der Kirchenprovinz Sachsen in Magdeburg war die entsprechende Akte nicht nachweisbar (nachrichtl. M. Hahn, 20.07.2011); Da bei den Recherchen in Betracht zu ziehen war, dass die Witwe nach dem Tod Flashars nach Sachsen-Anhalt zurückging, wären aufgrund möglicher Versorgungsansprüche der Witwe die Akten an die dortige Landeskirche überstellt worden. 
Palimpsesthandschriften posthum veröffentlichte ${ }^{20}$, schrieb: „Martin Flashar, der sich durch seine Arbeiten [...] als kenntnisreicher und gut beobachtender Septuagintaforscher eingeführt hatte, und von dessen Eifer die Septuaginta-Wissenschaft noch schöne Früchte hätte erwarten dürfen, ist leider am 22. Dez. 1914 als Offizierstellvertreter im 4. Garderegiment zu Fuß auf einem Patrouillengange bei Les Essarts im nördlichen Frankreich gefallen“ “. ${ }^{21}$

Sowohl Flashar als auch Duensing bezogen ein Stipendium für ihre Reise. Duensing erhielt sein Stipendium in Höhe von 1500 Mark vom Deutschen PalästinaVerein. Duensings Personalakte, die sich heute im Archiv der Braunschweigischen Landeskirche in Wolfenbüttel befindet, enthält ein Gesuch um zusätzliche finanzielle Unterstützung, das uns Aufschluss über die Kosten einer solchen Orientreise gibt und Einblick in die Reiseplanung gewährt. Die Flashar entstandenen Kosten dürften in etwa vergleichbar gewesen sein:

Pastor Duensing

Dassensen, den 16. Juli 1914

Betrifft Gesuch um Unterstützung zur Aufbringung der Kosten für seine Vertretung.

An das Kgl. Landeskonsistorium in Hannover

durch den Herrn Generalsuperintendenten in Hildesheim

und den Herrn Superintendenten in Einbeck.

In Ergänzung meines vor meiner Entsendung nach Jerusalem dem Königlichen Landeskonsistorium eingereichten Gesuches um Unterstützung zur Bestreitung der durch meine Vertretung entstandenen Kosten erlaube ich mir anbei einzusenden:

1. die Beträge der entstandenen Fuhrkosten, welche die Summe von 34 $M$ ergeben, und

2. eine Übersicht der von dem Spezialvikar sowie den beiden Lehrern in Dassensen und Wellersen geleisteten Vertretungsarbeit. Kommt für letztere der in Vertragzeiten übliche Satz in Anwendung, so ergiebt sich die Summe von 61,50 M. Eine Taxation der Arbeit des Spezialvikars, die an der Hand der eingereichten Übersicht möglich ist, möchte ich Kgl. Landeskonsistorium überlassen.

Zur genaueren Begründung meines Gesuches erlaube ich mir Folgendes nachzufügen. In den Mitteilungen und Ratschlägen für die Mitglieder des Institutes, welche zuerst 1905 im Palästinajahrbuch erschienen sind, seitdem

20 Rahlfs, Palimpsest-Fragmente.

21 Rahlfs, Palimpsest-Fragmente, 390 [406]. 
aber allen Mitgliedern als Sonderabzug gegeben worden, war von dem Vorsteher des Institutes folgende Berechnung angestellt. Von den $1500 \mathrm{M}$ des Stipendiums erfordert die direkte Hin- und Rückreise nach Palästina 600 M. Von den übrigbleibenden $900 \mathrm{M}$ beansprucht ein dreimonatlicher Aufenthalt in Palästina die Hälfte, 450 M. Das Hotel Fast in Jerusalem gebe den Mitgliedern volle Pension zu einem Vorzugspreis von 5 Francs für den Tag. Der Rest von $450 \mathrm{M}$ lasse sich dann für die Ausflüge des Institutes verwenden. - Diese Berechnung ist heute nicht mehr zutreffend; denn der Lebensunterhalt ist um volle 100\% gestiegen. Das Hotel Fast wollte uns jetzt einen Vorzugspreis von 9 bzw. 10 francs pro Tag geben. Wenn nun auch auf der andern Seite viel weniger wie $450 \mathrm{M}$ auf die Ausflüge zu rechnen ist, so reicht doch das Stipendium nicht mehr. Auch deshalb nicht mehr, weil nun Ansprüche an die Mitglieder gestellt werden, die früher nicht bestanden. So war man jetzt z. B. gezwungen, vorher eine Unfallversicherung einzugehen, die allein schon ca. $40 \mathrm{M}$ kostete. Dazu kommen noch die nicht in Anschlag gebrachten Ausrüstungskosten. Man muß also schon jetzt aus eigenen Mitteln zuschießen. Müßte man auch noch die ganze Vertretung tragen, so würde dieser Zuschuß erheblich verstärkt, und die Wohltat der Entsendung würde eine stark begrenzte werden.

Hugo Duensing.

Flashars Reisekosten hingegen waren anders gedeckt. Der Senat der FriedrichWilhelms-Universität hatte Flashar ein Jubiläumsstipendium der Stadt Berlin vermittelt. Flashars engere Bekanntschaft mit Wolf v. Baudissin (1847-1926), der 1900-1921 den Lehrstuhl für Altes Testament in Berlin inne hatte und 1912-1913 Rektor der Humboldt-Universität war, dürfte dabei nicht von Nachteil gewesen sein. Aufgrund der Gewährung des Stipendiums war Flashar gehalten, dem Senat einen Reisebericht vorzulegen. Dies tat Flashar im August des Jahres. ${ }^{22}$ Nach Flashars Tod gelangte der Bericht in den Besitz des Septuaginta-Unternehmens, wie Rahlfs in der bereits erwähnten Publikation der von Flashar bearbeiteten Palimpsesthandschriften anmerkt: „Durch gütige Vermittlung des Herrn Grafen Wolf v. Baudissin, der in Flashar einen ihm besonders werten Schüler betrauert, sind jedoch der Bericht und die Abschrift seit dem 13. Okt. 1915 dem SeptuagintaUnternehmen zu dauernder Aufbewahrung überwiesen". ${ }^{23}$ Seither befand sich der handschriftliche Bericht Flashars in der mit der Rahlfs-Nr. 406 versehenen Gießener Kapsel, die darüber hinaus noch Folgendes enthält:

22 Der handschriftliche Bericht trägt den Eingangsstempel vom 13. August 1914.

23 Rahlfs, Palimpsest-Fragmente, 390 [406]. 
- Eine Beilage zum Reisebericht Flashars

- Ein Kollationsheftchen zu Ra. 929

- Eine Postkarte Flashars an Rahlfs vom Juli 1914

- Zwei Sonderdrucke von Tisserants Artikel zu Ra. $406^{24}$

- Einen Sonderdruck aus Rahlfs' Kleinen Mitteilungen aus dem SeptuagintaUnternehmen von 1915

\section{Der Reisebericht Martin Flashars}

Der handschriftliche Reisebericht Flashars ist zweifellos ein besonderes Pretiosum, da wir über die Reisen des Septuaginta-Unternehmens ansonsten nur vage über die Jahresberichte des Instituts informiert sind. Der vorliegende Bericht ist ein wichtiges zeitgeschichtliches Dokument, das uns Informationen aus erster Hand über die Situation Palästinas im Jahr 1914 aus der Sicht eines deutschen Reisenden liefert. Die insgesamt 20 Seiten sind von späterer Hand mit blauem Buntstift auf der jeweiligen Recto-Seite von 52 bis 61 durchnummeriert. Das Papier hat das Format 33,0 $\times 21,0 \mathrm{~cm}$. Die Deckseite (f. 52r) trägt den Eingangsstempel: „Universität Berlin, Eing. 13 Aug. 1914“ sowie den Aktenvermerk: ,zu 2243 S.B. 1/14“. Ferner findet sich auf der ersten Seite die handschriftliche Bemerkung: „13. Okt. $1915 \mathrm{zu}$ dauernder Aufbewahrung beim Septuaginta-Unternehmen erhalten. A. Rahlfs“. Der Bericht beginnt mit f. 53r, wobei die Seiten von f. 53r an von der Hand Flashars fortlaufend am oberen Seitenrand paginiert sind. Es folgt eine Transkription des Berichtes, der, wo es dem Herausgeber angebracht erschien, zusätzlich mit kurzen Sacherläuterungen versehen ist.

24 E. Tisserant, Un manuscrit palimpseste de Job, in: RB 21 (1912), 481-503. 


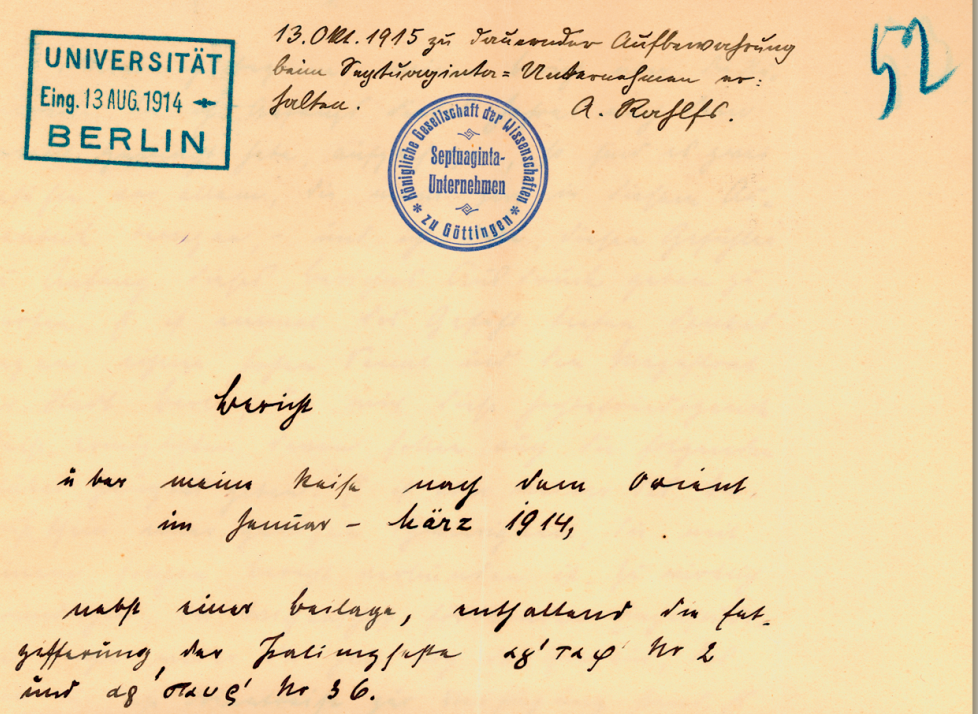

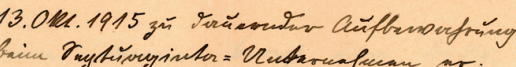
forltace.

Eing. 13 AUG. 1914 -

BERLIN

Septuaginta-

s 


\section{Bericht}

über meine Reise nach dem Orient im Januar - März 1914,

nebst einer Beilage, enthaltend die Entzifferung der Palimpseste

$\alpha \gamma^{\prime} \tau \alpha \varphi^{\prime}$ Nr. 2 und $\alpha \gamma^{\prime} \sigma \tau \alpha v \varrho$ ' Nr. 36.

lic. Dr. M. Flashar.

Wenn ich beginne, meinen Bericht über die Studienreise, die ich anfangs dieses Jahres nach dem Orient gemacht habe, aufzusetzen, so sind es zwei Gefühle vor allem, die mein Herz in diesem Augenblick bewegen, - und ich bitte, diesen Gefühlen zu Anfang dieses Berichtes Ausdruck geben zu dürfen. Es ist einmal das Gefühl tiefen Dankes gegen einen hohen Senat und den Magistrat der Stadt Berlin, die mir diese hochbefriedigende Reise ermöglichten. Davon sollen auch die folgenden Blätter Zeugnis geben. Es ist aber weiter das Bewusstsein einer gewissen Schwierigkeit, die mit einem solchen Bericht verbunden ist. Zu wirklich gründlichen, die Wissenschaft fördernden Einzelstudien reichte weder die Zeit noch das Geld aus, das mir für diese Orientreise zur Verfügung stand. Es kam hinzu, daß mein Amt mich nötigte, die Reise zu einer Zeit anzutreten, die für Palästina sehr ungünstig ist. Es war ein, für mich persönlich sehr glücklicher Zufall, daß in diesem Jahr die starken Winterregen ausnahmsweise fast ganz ausblieben, und es mir so möglich wurde, vielmehr vom Lande, und unter viel günstigeren Umständen zu sehen, als ich es gehofft hatte. Grade dies letzte schien mir aber eins der Hauptziele einer solchen Reise: in der kurz bemessenen Zeit ein möglichst umfassendes Bild von diesem Teil des Orients zu erhalten, von Land und Leuten, von seinem Einst und Jetzt. Damit scheint freilich gegeben, daß der Bericht nur Eindrücke wiedergeben und Erfahrungen erzählen kann, wie sie schon tausendfältig und viel besser dargestellt sind. Und doch lag für mich der größte Wert dieser Reise darin, daß sich der persönlichen Anschau[2]ung ein lebendiges Bild der Vergangenheit erschließt, wie es aus der Ferne kein Buch und keine Einbildungskraft geben kann. Wie unter einem Schleier birgt sich unter dem Leben und Treiben des heutigen Orients die Zeit, in der sich die Geschichte des alten und neuen Testaments abspielte. Auch sogleich enthüllt sie sich dem sich versenkenden Blick. So konservativ der Orient, selbst in den lächerlichsten Kleinigkeiten ist, so ist der erste Eindruck, den man oft erhält, der eines großen - Nichts. Nur es kommt hinzu, daß man das Land beladen mit einer Anzahl Vorurteilen und Erwartungen betritt, die man erst überwinden muß, um klar und richtig zu sehen. Es ist auch oft so, daß erst nachher der Wert der gesammelten Erfahrungen sich zeigt. Über manches Wort, über manche Erzählung las man ahnungslos hinweg. Nun bekommen sie ein[e] persönliche Note, einen ganz bestimmten Stimmungsgehalt, der aus der Erinnerung des selbst geschauten kommt. Ein Bericht kann das kaum wiedergeben. Er wird mehr 
das nüchterne Aufzählen von Orten sein, an denen ich gewesen. Ebensowenig wird er ein Bild von dem Erfolg der zweiten Aufgabe geben können, die ich mir stellte: Nämlich meine, auf der Universität erworbenen Kenntnisse des Arabischen durch hören und sprechen des heutigen Vulgärdialekts zu erweitern.

Es lag mir angesichts dieser Sachlage um somehr daran, auch ein greifbares, wissenschafliches Resultat von dieser Reise heimzubringen. Ich hatte mich zu diesem Zweck vorher an H. Professor Rahlfs in Göttingen gewandt, in dessen Händen die Leitung des großen SeptuagintaUnternehmens der Akademien der Wissenschaften zu Berlin, Göttingen und [3] Wien liegt. Er bezeichnete mir als besonders wertvoll und wichtig die Entzifferung zweier Palimpseste, die sich in der Patriarchalbibliothek

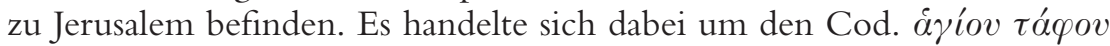
2, wahrscheinlich die älteste Minuskelhandschrift, die es giebt, in der auf fol. 27 und 56 unter dem Pentateuchtext der Anfang des Sirachbuches in Uncialschrift steht und zwar, nach Papadopoulos Kerameus ${ }^{25}$, geschrieben vielleicht schon im IV. Jahrhundert. Rendel Harris: Biblical fragments from Mt. Sinai. 1890. hat p. XIV diese Blätter beschrieben und zum teil, wenn auch nur flüchtig entziffert. ${ }^{26}$ - Der zweite Palimpsest befindet sich im Cod.

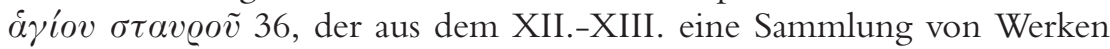
des Johannes Chrysestemus $>$ hl. Basilius $<$ enthält. Er ist vollständig palimpsest, und enthält in der unteren Schrift 1) verschiedene Werke des Johannes Chrysostomus, 2) den Text des Buches Hiob mit einer Katene des Olympiodorus Polychronius. E. Tisserant hat in der Revue Biblique. 1912, p. 481-503 diesen Hiobtext beschrieben und von 42 Blättern 161/2 gelesen. ${ }^{27}$

Die Ergebnisse meiner Arbeiten an diesen beiden Handschriften, über die ich an seinem Ort näher berichten werde, liegen dem Bericht gesondert bei.

Ich trat meine Reise am 1. Januar dieses Jahres an, nachdem mir der Ev. Ober Kirchenrat einen zehnwöchentlichen Urlaub bewilligt hatte. $\mathrm{Zu}-$ nächst fuhr ich nach Göttingen, um mit Herrn Professor A. Rahlfs nochmals Rücksprache zu nehmen. Ich lernte bei dieser Gelegenheit die Grundzüge und das Material des großen Septuagintaunternehmens näher kennen. Herr Professor Rahlfs war so gütig, mir noch ein besonderes Empfehlungsschreiben seitens des Vorstandes des S.-U. [4] mitzugeben, und forderte

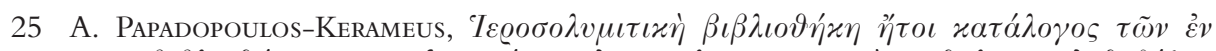

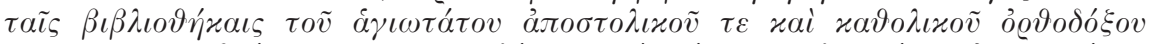

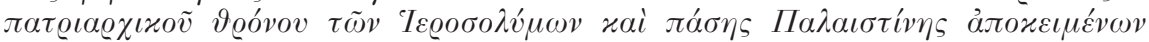

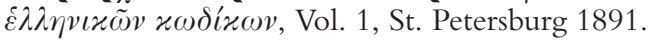

26 J. R. Harris, Biblical Fragments from Mount Sinai, London 1890. Zu James Rendel Harris (1852-1941) vgl. C. Bernet, Art. „Harris, James Rendel“", in: BBKL 30, Nordhausen 2009, 557-569.

27 Tisserant, Un manuscrit palimpseste de Job. Zu Eugène Tisserant (1884-1972) vgl. J. KreuzenbeCK, Art. „Tisserant, Eugène“, in: BBKL 20, Nordhausen 2002, 1463-1466. 
mich auf, gemeinsam mit Herrn lic. Dr. Duensing, der gleichzeitig in Jerusalem Photographien sämtlicher Septuagintahandschriften besorgen sollte, beim Patriarchat vorzugehen.

Über Mailand - Genua - Pisa fuhr ich dann nach Neapel, mit einigem Aufenthalt an den genannten Städten. So wertvoll mir die Eindrücke dieser kurzen Tage sind, darf ich sie wohl in diesem Bericht übergehen. Am 9. Januar schiffte ich mich in Neapel auf dem „Prinzregent Luitpold“"28 ein, und kam am 12. in Alexandrien an. Ich habe meinen Aufenthalt in Agypten auf Cairo beschränkt, weil ich nur wenige Tage auf Agypten verwenden und diese Zeit nicht unnötig zersplittern wollte. Auch so konnte ich in Cairo, - von kurzen Ausflügen nach Gizeh, Sakkarah ${ }^{29}$ und Heliopolis ${ }^{30}$ abgesehen, nur die wichtigsten Baudenkmäler sehen und täglich einige Stunden dem Museum, dieser einzigartigen Sammlung ägyptischer Kulturdenkmäler, widmen. Leider war[en] der Besuch und das Studiums des Museums dadurch erschwert, daß dieses wieder einmal vor kurzem umgezogen war. Manche Säle waren überhaupt unzugänglich und vielfach fehlten die Signaturen. Angesichts der überwältigenden Fülle gleichartiger Gegenstände war mir der ausgezeichnete Guide du visiteur au musée du Caire par G. Maspero. 1914. besonders wertvoll. ${ }^{31}$

So eigenartig und fesselnd der Gegensatz zwischen uralter und hypermoderner Kultur, zwischen dem Luxus der europäischen Stadtteile und der trostlosen Verkommenheit der orientalischen, zwischen den altersgrauen Pyra[5]miden und dem Flugzeug, das sie umsurrt, ist, so sind der unerfreulichen und unbefriedigenden Züge im modernen Ägypten doch zu viel, als daß ich nicht mit einiger Befriedigung am 18. Januar dies merkwürdige Land wieder verließ. Was übrigens die - kaum sichtbare - englische Regierung für die materielle Hebung des Landes getan hat, wird dem Reisenden erst klar, wenn er Palästina und damit ein typisches Beispiel türkischen Regimes kennen gelernt hat.

Da ich annahm, in Palästina in die übliche Regenzeit hineinzukommen $^{32}$, hatte ich vor, zunächst einige Wochen in Jerusalem zu bleiben. Es war das eine unnötige Vorsicht, denn im Gegensatz zu früheren Jahren hat es in Jerusalem in diesem Winter vom 4. Advent bis Mitte Mitte Februar

28 Die Prinzregent Luitpold war ein Dampfschiff der Norddeutschen Lloyd, das vor dem 1. Weltkrieg auf der Linie zwischen Marseille, resp. Genua und Alexandria verkehrte.

29 Die altägyptische Nekropole Sakkarah befindet sich $20 \mathrm{~km}$ südlich von Kairo.

30 Grabungen in der nordöstlich von Kairo gelegenen „Sonnenstadt“ erfolgten vor dem 1. Weltkrieg in den Jahren 1903-1904 unter Ernesto Schiaparelli (1856-1928) und 1912 unter Flinders Petrie (1853-1942).

31 G. Maspero, Guide du visiteur au Musée du Caire, Kairo ${ }^{3} 1914$.

$32 \mathrm{Zu}$ den klimatischen Bedingungen des Frühlings und dem typischen Frühlingsregen in Palästina vgl. G. Dalman, Arbeit und Sitte in Palästina, Vol. 1: Jahreslauf und Tageslauf. 2. Hälfte, Frühling und Sommer (SDPI 3/2, zugleich BFChTh, II/15), Gütersloh 1928, 281-468, bes. 291-304. 
nur an einigen ganz wenigen Tagen geregnet, so daß sich gegen Ende meines Aufenthaltes schon die bedenkliche Calamität einer Wasserversorgung bemerkbar $>$ machte $<$, bei der eine größere Stadt fast ausschließlich auf Regenwasser angewiesen ist. Immerhin hatten meine Dispositionen den großen Nachteil, daß mein Kollege, Herr lic. Duensing, wider Erwarten erst Anfang Februar nach Jerusalem kam und sogleich mit dem archäologischen Institut auf fünf Tage nach Jericho ging. Da Herr Generalkonsul Schmidt ${ }^{33}$ vorschlug, wir sollten gemeinsam unseren Besuch beim griechischen Patriarchen machen, um Erlaubnis zum Photographieren und Lesen der Handschriften zu erhalten, mußte ich bis zum 8. Februar mit diesen Arbeiten warten. An diesem Tage fand unser Besuch bei „Sa Béatitude“ statt und dank [6] der liebenswürdigen und energischen Befürwortung durch den Herrn Generalkonsul wurde uns die Erfüllung all unserer Wünsche bereitwillig zugestanden.

Die Bibliothek des griechischen Patriarchats liegt auf dem Dach des großen Klosters, - es war jedesmal eine schwierige Reise durch all die Vorgänge, Treppen und Höfe hindurch. Es giebt in ganz Jerusalem kaum ein so kompliciertes, merkwürdiges Gebäu[de], wie dies Kloster. Ebenso merkwürdig die Bibliothek selbst, mit einem Arbeitsraum, der etwa $3 \mathrm{~m}$ breit und $7 \mathrm{~m}$ lang ist, - und dazu noch sein Licht aus Kerzen bekommt. Um so großartiger sind die handschriftlichen Schätze, die diese höchst dürftigen und feuergefährlichen Zimmer bergen.

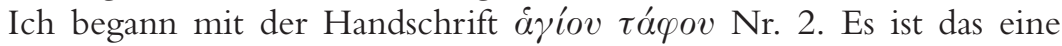
prachtvolle Handschrift des Oktateuch, vielleicht eine der ältesten Minuskelhandschriften, die es giebt. Zwei ihrer Blätter sind palimpsest, nämlich fol. 27 und 56, und enthalten Sirach, Prolog Z. 12. bis Ende und I,1 III,2. Die ältere Schrift, eine wunderbare regelmäßige und formenschöne Majuskel, die möglicherweise aus dem IV. Jahrhundert stammt, war an manchen Stellen deutlich zu lesen, an anderen Stellen war sie sehr sorgfältig abgewaschen und von der darüberliegenden Schrift verdeckt. Hier blieb nichts übrig, als Schwefelammonium zuhilfe zu nehmen, was von Mühe und fürchterlichem Geruch abgesehen, stets eine gefährliche Sache

33 Dr. Edmund Schmidt (1855-1916) war von 1901 bis 1916 Konsul in Jerusalem. - Die Berichte des (zwischen 1918 und 1924 geschlossenen) deutschen Generalkonsulats in Jerusalem finden sich heute im Politischen Archiv des Auswärtigen Amtes in Berlin und sind bis 1936 nahezu vollständig erhalten. 1955 erwarben die Israelischen Staatsarchive in Jerusalem die Konsulatskorrespondenz der Jahre 1839-1939, die heute unter der Signatur G.C., R.G. 67 (Israel State Archives, German Consulate, Record Group 67) verwahrt werden. Zu E. Schmidt vgl. ausführlicher: M. Eliav (ed.), Die Juden Palästinas in der deutschen Politik. Dokumente aus dem Archiv des deutschen Konsulats in Jerusalem, 1842-1914, Tel Aviv 1973, XVI; Z.W. SAdmon, Die Gründung des Technions in Haifa im Lichte deutscher Politik 1907-1920 (EHKB 78), München 1994, 23; S. Wulf, Jerusalem, Aleppo, Konstantinopel. Der Hamburger Tropenmediziner Peter Mühlens im Osmanischen Reich am Vorabend und zu Beginn des Ersten Weltkriegs (Hamburger Studien zur Geschichte der Medizin 5), Münster 2005, 20 Anm. 66. 
ist, wenn die obenliegende Schrift nicht verdorben werden soll. ${ }^{34}$ Ich prüfte zu nächst den Teil der Blätter nach, den Rendel Harris schon gelesen und veröffentlicht hat. Stichproben zeigten, daß Ren[7]del Harris vielfach grade an wichtigen Stellen die Lesart der Septuagintaausgabe, die er bei seiner Arbeit zur Hand hatte, in den Palimpsest hineingelesen hat, sogar an Stellen, wo dieser ziemlich deutlich zu lesen ist. Es war daher nötig, den ganzen Text noch einmal zu lesen. Ich habe dabei an 37 Stellen festgestellt, daß Rendel Harris' Veröffentlichung ungenau oder falsch ist. Die Vergleichung dieses Textes sowie des neu gelesenen Teils mit den anderen Textzeugen ergiebt, daß der Palimpsest einen Texttypus darstellt, der von den übrigen Uncialen nicht unwesentlich abweicht, aber mit einer Gruppe von $4 \mathrm{Mi}$ nuskeln übereinstimmt. Durch diese Übereinstimmung wird erwiesen, daß dieser Texttypus schon einer sehr alten Zeit angehört. Die eingehenderen Untersuchungen über diesen Texttypus gehören nicht in diesen Bericht. ${ }^{35}$

War die Entzifferung dieses Palimpsestes zwar schwierig aber immerhin noch möglich, so gestaltete sich die Lesung der zweiten Handschrift, $\alpha \gamma$ íov

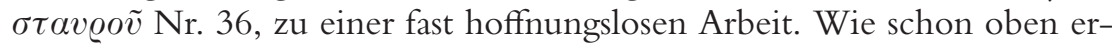
wähnt, enthält dieser Codex eine Sammlung von Werken des h. Basilius. $\mathrm{Zu}$ seiner Herstellung wurden Blätter zweier älterer Handschriften verwendet, nämlich 1) eine Sammlung von Werken des Johannes Chrysostomus und 2) ein Exemplar des Buches Job mit einer Catene des Olympiodorus Polychronius. Mir kam es ausschließlich auf den Jobtext an, den zum Teil Tisserant veröffentlicht hat. Wie Stichproben zeigten, hat Tisserant se mit großer Sorgfalt und Genauigkeit gearbeitet. Dafür hat er sich auch auf die Blätter beschränkt, die einigermaßen les[8]bar sind. Bei den übrigen 26 Blättern lässt sich zum größten Teil nur aus der Art des Pergaments erken-

34 Schwefelammonium, das auch als „Flüchtige Schwefelleber“ bezeichnet wird, fand vornehmlich in der Palimpsestforschung des 19. Jh.s Verwendung. Bei diesem Reagenz handelt es sich um eine Ammoniumhydrogensulfid-Lösung $\left(\mathrm{NH}_{4} \mathrm{HS}+\mathrm{H}_{2} \mathrm{O}\right)$. Es beruht auf dem Prinzip, dass die metallischen Spuren der abgewaschenen Tinte der scriptio inferior (d.h. der „,unteren Schrift") durch den Kontakt mit der Sulfid-Lösung als Sulfide ausfallen und dadurch zu einem optischen Auffrischen der alten Tintenreste beitragen. Die in den verbliebenen Tintenresten vorhandenen Kationen von Magnesium Nickel, Kobald, Zink $\left(\mathrm{Mn}^{2+}, \mathrm{Ni}^{2+}, \mathrm{Co}^{2+}, \mathrm{Zn}^{2+}\right)$ usw. werden als entsprechende Sulfide gefällt (MgS, NiS, $\mathrm{CoS}, \mathrm{ZnS}$ ). Ein unangenehmer Nebeneffekt der Anwendung jenes Reduktionsmittels ist die Bildung von gasförmigem Schwefelwasserstoff $\left(\mathrm{H}_{2} \mathrm{~S}\right)$, was den typischen Geruch nach faulen Eiern hervorruft.

Zur Anwendung chemischer Reagenzien in der Palimpsestforschung des 19. Jh.s vgl. F. Albrecht, Between Boon and Bane. The Use of Chemical Reagents in Palimpsest Research in the Nineteenth Century, in: Care and Conservation of Manuscripts 13, Kopenhagen 2012, 147-165.

35 Zum Texttyp von Ra. 929 vgl. J. ZIEgler, Sapientia Jesu Filii Sirach (Septuaginta. Vetus Testamentum Graecum Auctoritate Academiae Scientiarum Gottingensis editum XII/2), Göttingen ${ }^{2}$ 1981, 56, der diese Hs. im Sirachbuch der Gruppe $(a)=$ Ra.-Hss. 149-260606 zuordnet: „,Zu dieser Gruppe gehört auch das Fragment 929“. Über die Gruppe (a) sagt Ziegler, dass sie „oftmals von der lukianischen Rezension $(L)$ abhängig“ sei (ebd. 70). 
nen, daß sie zum Jobexemplar gehört haben müssen, von der Schrift war vielfach kaum eine Spur zu sehen. Ich war infolgedessen fast ausschließlich darauf angewiesen, mit Schwefelammonium zu arbeiten. Aber auch dies versagt an vielen Stellen, nämlich wenn die jüngere Schrift genau über der alten liegt. Es ist außerdem ein so zeitraubendes Verfahren, daß man stundenlang an einer einzigen Zeile sitzt. Besonders erschwert wurde diese Arbeit auch dadurch, daß mir als einziges Hilfsmittel der 1. Teil der alten Septuagintakonkordanz von Tromius zur Verfügung stand, und auch dieser von Würmern derartig zerfressen, daß manche Blätter vollkommen auseinanderfielen. ${ }^{36}$ Ehe ich ein Blatt identificiert hatte, vergingen Tage, so daß ich trotz aller Mühe nur 15 Zeilen habe lesen können. Ich mußte schließlich die Arbeit, wenn auch unvollendet aufgeben, da ich meine beschränkte Zeit auf Dinge verwenden wollte, die mir schließlich wertvoller waren, als dieser Text. -

Während dieses längeren Aufenthalts in Jerusalem war es mir ein Hauptanliegen, eine genaue Kenntnis der Topographie und Baugeschichte der Stadt zu gewinnen. Wertvoll war mir in dieser Hinsicht die reichhaltige Bibliothek des Jer[usalemer] Johanniterhospizes ${ }^{37}$, in dem ich wohnte. Viel verdanke ich auch der sachkundigen Führung Herrn Prof. Dalmans. ${ }^{38}$ Mit ihm und dem archäolog. Institut besuchte ich z.B. mehrmals den Tempelplatz, den Lauf der jetzigen und die ausgegrabenen Reste der früheren Stadtmauern, Siloaskanal, Grabeskirche, Be[9]tsasda (St. Anne) u.a. Bei Prof. Dalman nahm ich auch an den Vorlesungen des Instituts über Arbeit und Sitte der Fellachen teil, in denen während meines Daseins der Ackerbau nach Bodenverhältnissen und Klima, Sitten und Werkzeugen eingehend besprochen wurde. ${ }^{39}$ Die Beobachtungen draußen vor der Stadt, wo grade die Bestallung des Landes vor sich ging, gaben dazu eine lebendige Illustration.

Daß ich im Johanniterhospital wohnte, war mir auch deshalb lieb, weil es mitten in der Altstadt, an der stets dicht bevölkerten Markt,,straße“ liegt. Ich hatte so täglich Gelegenheit das Treiben der Handwerker, das Gebahren von Käufer und Verkäufer zu beobachten und im Gespräch mit den Eingeborenen meine Sprachkenntnisse zu vermehren. Grade dies ist eigentlich

36 A. Tromm, Concordantiae Graecae in Septuaginta Interpretes. 2 Vol., Amstelodami/Trajecti ad Rhenum 1718.

37 Zum Jerusalemer Johanniterhospitz vgl. J. EISLER, Das Johanniterhospiz in Jerusalem, Berlin 2008. Das Hospiz des Johanniterordens befindet sich seit 1866 in der Via Dolorosa.

38 Zu Prof. Dr. Gustaf Dalman (1855-1941) vgl. J. MännChen, Gustaf Dalmans Leben und Wirken in der Brüdergemeinde, für die Judenmission und an der Universität Leipzig 1855-1902 (ADPV 9/1), Wiesbaden 1987; J. Männchen, Gustaf Dalman als Palästinawissenschaftler in Jerusalem und Greifswald 1902-1941 (ADPV 9/2), Wiesbaden 1991.

39 Den Ackerbau Palästinas hat Dalman späterhin monographisch abgehandelt, vgl. G. DALMAN, Arbeit und Sitte in Palästina, Vol. 2: Der Ackerbau (SDPI 5, zugleich BFChTh, II/27), Gütersloh 1932. 
das wichtigste, was man an Vorkenntnissen in das Land mitbringen muß; ohne das geht man, vor allem außerhalb der großen Städte leer aus.

So war es neben dem alten auch das neue Jerusalem, das ich kennen zu lernen suchte. Vor allem die deutschen Anstalten: die deutsche Schule, das syrische Waisenhaus, Aussätzigenhospital und Talitha kumi, das Kaiserswerther Diakonissen-Krankenhaus und die Ölbergstiftung. ${ }^{40}$ Es ist ein bedeutendes Stück deutscher Kultur- und Missionsarbeit, das ich hier kennen lernte, wie überhaupt das Deutschtum grade in Jerusalem allen anderen Nationen gegenüber hervortritt. Den größten Eindruck davon hatte ich bei der Kaisergeburtstagsfeier. ${ }^{41}$ Beim Empfang im Generalkonsulat und noch mehr bei der Nachmittagsfeier im [10] katholischen Paulus-Hospiz trat das hervor: Man traf dort den Franziskaner auf dem Platz neben dem deutschen Juden im langen Kaftan und mit wehenden Locken, die Schwaben aus der Templerkolonie neben den deutschen Beamten und Kaufleuten, eine Kolonie, wie sie keine andere Nation aufbringen kann, imposant in ihrer Zahl und Geschlossenheit, wie sie auch in den Festreden zum Ausdruck kam. Daß hiervon auch die Wirte des Tages, die Lazaristenpatres ${ }^{42}$ keine Ausnahme machten, obgleich sie in Deutschland selbst verboten sind, war mir besonders bemerkenswert. - Eigentümlich ist der Eindruck, den man von den Templern erhältt. ${ }^{43}$ Aesthetisch und vom wirtschaftlichen Standpunkt aus macht ihre Kolonie draußen vor dem Bahnhof einen prächtigen Eindruck. Im Inneren bieten sie dagegen das Bild einer Sekte, deren religiöse Ansätze abgestorben sind, so daß die bloße Negation übrig bleibt. Wie man mir sagte, soll auch ihre kaufmännische Moral hie und da darunter leiden; ich kann dafür aber in keiner Weise einstehen. Teilweise haben sie den Anschluß an die reichsdeutsche Kolonie und die evangelische Gemeinde

40 Zum Aussätzigenhospital vgl. N. Schwake, Die Entwicklung des Krankenhauswesens der Stadt Jerusalem vom Ende des 18. bis zum Beginn des 20. Jahrhunderts (Studien zur Geschichte des Krankenhauswesens 8), Herzogenrath 1983, 603-634 und zum DiakonissenKrankenhaus ebd. 245-346; 681-692. Zu Talitha kumi vgl. A. Nothnagle (ed.), Seht, wir gehen hinauf nach Jerusalem. Festschrift zum 150jährigen Jubiläum von Talitha Kumi und des Jerusalemvereins, Leipzig 2001. Zur Ölbergstiftung vgl. J. EIsLER, Die AugusteVictoria-Stiftung auf dem Ölberg in Jerusalem. Eine Bilddokumentation (Kleine Schriften des Vereins für württembergische Kirchengeschichte 8), Stuttgart 2010 und H. WawrZYN, Ham and Eggs in Palestine. The Auguste Victoria Foundation 1898-1939, Marburg 2005.

41 Die kaiserliche Geburtstagsfeier für Friedrich Wilhelm II. (1859-1941) wurde am 27. Januar begangen.

42 Die Kongregation der Lazaristen (Congregatio Missionis) wurde 1873 im Rahmen des Kulturkampfes in Deutschland per Gesetz verboten; vgl. Deutsches Reichsgesetzblatt 1873, Nr. 12, 109.

43 Zur Templerkolonie vgl. ausführlich A. CARmel, Die Siedlungen der württembergischen Templer in Palästina 1868-1918. Ihre lokalpolitischen und internationalen Probleme. Aus dem Hebräischen übersetzt von P. Leshem (Veröffentlichungen der Kommission für geschichtliche Landeskunde in Baden-Württemberg. Reihe B, Forschungen 77), Stuttgart ${ }^{3} 2000$. 
wieder gefunden. Es ist das wohl hauptsächlich das Verdienst zweier Persönlichkeiten: des Generalkonsuls Schmidt und des Propstes Dr. Jeremias. ${ }^{44}$ Nebenbei, wie das bei Deutschen ja wohl nicht anders sein kann, bildet Faicks Bierstube ${ }^{45}$ ein starkes Einigungsmoment. An jedem Montag ist dort Jung und Alt, vom Generalkonsul bis zum letzten Schwaben versammelt, um für ein „Blechle“ zu - kegeln.

Von großem Interesse ist augenblicklich nicht nur in Jerusalem, sondern in Palästina über[11]haupt die Judenfrage. ${ }^{46}$ Die zionistische Bewegung gewinnt ständig an Umfang und Bedeutung. Während die ältere Generation noch deutsch bez[iehungs]w[eise] Jiddisch sprach, lernt und spricht die Jugend nur Hebräisch, - der Streit, der sich in den deutsch-jüdischen Hilfsschulen darüber erhob, hat ja auch in Deutschland viel Aufsehen gemacht. Von der Bedeutung dieser national jüdischen Bewegung, - ob sie wirklich überall national ist, steht dahin, - bekam ich später auf meiner Reise nach dem Norden einen lebhaften Eindruck. In Nabuhrs lernte ich einen New Yorker Millionär Rosenbaum kennen, der umfangreiche Ländereien kaufen wollte, und ebenso ist in Galiläa, in der Umgebung des Tabor, auf der Hochebene bei Tiberias und am See Genezareth ungeheuer viel Land in jüdischen Besitz gekommen. Während ich in Jerusalem war, erlebte ich den Einzug eines der Barone Rothschild. ${ }^{47}$ Tausende von jüdischen Schulkindern und Vereinen bildeten Spalier, als er in Begleitung türkischen Militärs vom Bahnhof zur Stadt fuhr, empfangen von den Rabbinern und einer Ehrenpforte mit den Worten ברוך הבא ,Gelobt sei der da kommt.“ Er hat dann auch, wie man in Jerusalem erzählte, 100 Millionen in Ländereien angelegt. Übrigens sprach man davon, daß die türkische Regierung entschlossen sei, dem Anwachsen der zionistischen Bewegung einen Riegel vorzuschieben. Ob ihr das gelingen wird, steht dahin. Jedenfalls sind die Juden der Ansicht, wie ich gesprächsweise hörte, daß die großen Landkäufe eine ausgezeichnete Spekulation sind. Das Land ist infolge der Verarmung der Fellachenbevölkerung und des unerträglichen Steu[12] erdrucks billig, während man andrerseits bestimmt damit rechnet, daß in absehbarer Zeit

44 Dr. Friedrich Jeremias (1868-1945) war von 1910-1914 Propst der ev.-luth. Erlöserkirche in Jerusalem. Bekannter ist sein Sohn, der Göttinger Neutestamentler Joachim Jeremias (1900-1979).

45 „Faicks Bierstube“ ist nicht näher zu lokalisieren.

$46 \mathrm{Zu}$ den beiden ,Nationalismen' Zionismus und Arabismus in der Zeit vor dem 1. Weltkrieg vgl. G. KrÄMer, Geschichte Palästinas. Von der osmanischen Eroberung bis zur Gründung des Staates Israel (Beck'sche Reihe 1461), München ${ }^{2} 2002$, 122-153; 390-393.

47 Baron Edmond de Rothschild (1845-1934) hatte zuvor schon dreimal Palästina besucht (1887; 1893; 1899). Im Februar 1914 fand sein vierter Besuch statt. - Zu Edmond de Rothschilds Engagement im Rahmen der zionistischen Bewegung vgl. Y. MAYOREK, Zwischen Ost und West. Edmond de Rothschild und Palästina, in: G. Heuberger (ed.), Die Rothschilds. Vol. 2, Beiträge zur Geschichte einer europäischen Familie, Sigmaringen 1994, 133-150. Zeitgenössische Photographien des vierten Besuches finden sich ebd. 139 u. 141 . 
das Land in anderen, vermutlich englischen Besitz übergeht und dann einen ähnlichen Aufschwung erlebt, wie Ägypten. Mit dem Anwachsen des jüdischen Landbesitzes hängt es zusammen, daß die Templer, z.B. in Jaffa, anfangen, sich beengt zu fühlen, und mit dem Gedanken umgehen, in das Gebiet der Bagdadbahn abzuwandern.

Ich gebe im Folgenden einen kurzen Überblick über die weiteren Exkursionen, die ich von Jerusalem aus gemacht habe.

Am 22. Januar ritt ich mit Herrn Prof. Dalman nach Ramallah. Der Weg (die breite Nabluser Fahrstraße) führte uns zunächst an der Wasserscheide entlang. Links wurde Scha'fât (in dem man Nob vermutet hat), dann rechts der Tell el-Fûl sichtbar, dies dem Gibea Benjamins ${ }^{48}$ entsprechend. Wichtiger war der etwa $45 \mathrm{~min}$. nördlich von a-Ram gelegene Hügel Tell en-naşbe. Der Weg windet sich dort in einem verhältnismäßig engen Tal zwischen zwei Hügeln hindurch. Da der Tell zudem eine ziemlich ergiebige Quelle besitzt (jetzt in einem jämmerlichen Chan $^{49}$ unten an der Brücke verborgen), so ist mit Sicherheit anzunehmen, daß in alter Zeit hier eine befestigte Ortslage gewesen ist, die vorzüglich geeignet war, den Weg zu sperren. Nach Prof. Dalmans Meinung ist hier Mispa zu suchen. Die Berichte $1 \mathrm{Kg} .15$ und Jerem. 41 passen ausgezeichnet auf diese Ortslage, fordern jedenfalls, daß Mispa an einer beherrschenden Stelle unmittelbar an de[r] gro[13]ßen Nord-Südstraße lag. Das oft für Mispa genannte Neti Samwîl kann keinesfalls in Betracht kommen, wie man sich leicht bei einem Besuch an Ort und Stelle überzeugen kann.

Kurz vor el Bîre (= Beeroth?) bogen wir links ab und ritten auf einem Höhenzug dem großen Dorf Ramallah zu. Die Einwohner sind fast durchweg (griechische) Christen. Die Frauen tragen neben einer bunten, kleidsamen Tracht einen eigenartigen Kopfschmuck: über einem Tuch zwei dicke Wülste aus großen Silbermünzen, ähnlich den schwarzen Tücherwülsten, mit denen die Männer in Syrien allgemein ih[re] keffije ${ }^{50}$ festhalten. Wir ritten durch das Dorf hindurch in eins der nach Norden liegenden steil abfallenden Täler um auf den terassenartig angelegten Feldern - Blumen zu suchen. Es blühen hier an ihrem südlichsten Punkt die schönen blauen und lila Anemonen, während man bei Jerusalem nur purpurrote findet. - Auf dem Rückweg kehrten wir bei einem Fellachen namens Chelît ein. Äußerlich von den anderen nicht zu unterscheiden, war er schon zweimal längere Zeit in Amerika gewesen. Die Auswanderung der jungen Leute ist überhaupt erstaunlich groß. In manchen Dörfern sieht man außer den Frauen nur Kinder und alte Männer. Der Grund liegt einmal in der Furcht vor dem Militärdienst, - zuweilen soll die Dienstzeit auf 9 Jahre ausgedehnt werden,

48 Vgl. Ri 19f.

49 Der „Chan“ bezeichnet im Vorderen Orient eine Herberge.

50 Die keffije ist ein buntes Seidentuch. Zu den gebräuchlichen Kopfbedeckungen der damaligen Zeit vgl. G. Dalman, Arbeit und Sitte in Palästina, Vol. 5: Webstoff, Spinnen, Weben, Kleidung (SDPI 8, zugleich BFChTh, II/36), Gütersloh 1937, 251-257. 
- zum anderen in der Unmöglichkeit, unter der türkischen Herrschaft auch nur zu bescheidenstem Wohlstande zu gelangen. - Merkwürdig ist nur, daß wie man sagt die Auswanderer fast ausnahms[14]los zurückkehren, sobald sie sich ein paar tausend Francs erspart haben. - Den Rückweg nahmen wir durch das Wadi ed-Der, an Ed-Dschib, dem alten Gibeon vorbei, das auf einem merkwürdigen, in lauter kreisförmigen Terrassen aufsteigenden Hügel liegt.

Am 24. Januar ritt ich mit einem deutschen Ingenieur von der Bagdadbahn nach Jericho. Wir besichtigten eingehend Vellins Ausgrabungen auf dem Tell in der Nähe der Sultansquelle, ritten dann zum unteren Eingang des Wadi Kelt, um das griechische Strafkloster zu besuchen, das oben im Wadi in schauerlicher Einöde liegt. ${ }^{51}$ Am nächsten Tage besuchten wir das tote Meer und die sogenannte Taufstelle bei nicht weit von dem Dir Mar Juhannā.

Am 30. Januar ritt ich zusammen mit dem Referenten Dr. Grobber vom deutschen Generalkonsulat in die Gegend südlich von Jerusalem. Wir ritten zunächst an Bethlehem und Bet Dschala vorüber nach den sog. SalomonsTeichen, el Burak, frei in den Felsen eingehauenen rechtwinkligen Becken von ziemlich bedeutenden Dimensionen. Von hier ging es an den kleinen, aber von einem sehr stattlichen Kloster ${ }^{52}$ beherrschten Muslimendorf Arțas vorbei, auf halsbrecherischen Wegen durch das Wadi gleichen Namens zum Wadi Charêtûn hinüber, mit alten Befestigungen und Einsiedlerhöhlen in wild romantischer Gegend. Interessant war der Besuch der Marâret Ch[arêtûn], der sog. Höhle Adullam, in die wir, geführt von einigen höchst zweifelhaften Beduinen hinein krochen. Wenn auch [15] schwerlich der Höhle Adullam ${ }^{53}$ gleichzusetzen, giebt sie doch mit ihrem unzugänglichen, leicht zu sperrenden Eingang ein lebhaftes Bild von den Löchern und Felsenritzen, in denen sich Israel so oft verkroch. - Von dort gings in brennender Mittagshitze zum Dschebel el Furêdîs empor, heute meist Frankenberg genannt. Hier lag die von Herodes dem Gr. errichtete Festung Herodeion, von der außer Spuren am Fuße des (künstlich aufgeschütteten?) Bergkegels noch Reste der Mauer und der vier runden Türme auf dem Gipfel erhalten sind. Die Aussicht ist besonders nach Osten und Norden, wunderbar schön. - Den Rückweg nahmen wir direkt über Bethlehem, das hier, nach Süden zu, ungeheuer steil abfällt[.]

51 Bei dem genannten Kloster handelt es sich um das in der Spätantike gegründete Georgskloster, das im 12. Jh. von Manuel I. Komnenos (1118-1180) instandgesetzt wurde, in späterer Zeit verfiel und im 19. Jh. (1878-1901) durch griechische Mönche wiedererrichtet und neubesiedelt wurde.

52 Das Nonnenkloster „Hortus Conclusus“ wurde 1894 vom italienischen Orden der Sorelle di S. Maria del Giardino gegründet.

53 Die Höhle Adullam diente David als Zufluchtsort; vgl. 1.Sam 22,1; 2.Sam 23,13; 1.Chron 11,15 . 
Bethlehem besuchte ich besonders zwei Tage später, am 2. Februar, sowie am 3. Februar Ain Karim und auf dem Wege dorthin das festungsähnliche Kloster Dir il Musallibe. ${ }^{54}$

Am 7. Februar: Ausflug (mit Fr. v. Nathusius zusammen) nach Michmas, über el Bire und Batîm, der Gegend des alten Bethel, wo die verschiedenen Trümmer besichtigt wurden, ebenso wie in Dêr Diwan, in dessen Nähe das alte, als Festung ungleich wuchtigere Ai zu suchen ist. Von Michmas ritten wir dem hohen Ras et-țawîl <zu> und trafen hier Dalman mit dem archäologischen Institut, die vom Jordan hinaufkamen. Auf dem Rückweg wurde in Michmas die Philisterschlacht 1 Sam 14. besprochen, von der man angesichts der Szenerie ein außerordentlich klares Bild bekommt, eigentlich noch mehr, <wenn> man von dem ungleich höhergelegenen Dscheba' auf der durch ein tiefes [16] Tal von Dscheba' getrennte Michmas herabsieht. Von Michmas ritten wir sodann quer durch das wilde steinige Gebirge den „Weg“, den nach Jes. 10 die Assyrer gegen Jerusalem gezogen sind.

Am 8. Februar Ausflug nach Mar Saba ${ }^{55}$ im prachtvollen Wadi en-Nār und dem Berg el kuntâr mit schöner Aussicht auf das tote Meer. Besonders der Rückritt bei Vollmondschein gehört zu dem Schönsten, was ich in Palästina erlebt habe.

Am 16. Februar ritt ich über Bet Hanina nach En Nebi Samwîl, der die ganze Umgebung von Jerusalem beherrschenden Warte, die aber trotzdem schauerlich mit dem alten Mispa gleichzusetzen ist. Die Aussicht giebt ein überaus klares Bild von der geographischen Gliederung des Landes. Man sieht das tote Meer, ebenso wie das Mittelmeer und den breiten Küstenstreifen mit Jaffa und er-Ramle. Klar tritt der Gebirgszug der Moabiterberge und Gileads hervor, davor die Wasserscheide, die man von Jerusalem bis zu den Bergen bei Nabulus überblickt. Sehr merkwürdig ist unten im Tale der Anblick von Ed Dschib mit seinem eigenartigen Terrassenhügel - den Rückweg nahm ich über Bet Iksa und Kalonije an der Jaffastraße.

Am 18. Februar Ausflug über Anāta, das alte Anathoth, nach dem Wadi Fara, an dessen starken Quellen ein russisches Kloster mit uralten in die senkrechten Felsen gehauenen Höhlen liegt. ${ }^{56}$ Rückweg über Dscheba' und Er-Ram. Dies war der letzte größere Ausflug, den ich von Jerusalem aus machte.

54 Das von Flashar als „festungsähnlich“ beschriebene seit dem ausgehenden 18. Jh. griechisch-orthodoxe Kreuzkloster ist eine ursprünglich georgische Gründung.

55 Das griechisch-orthodoxe Kloster Mar Saba wurde 439 gegründet und gehört zu den bedeutendsten Klöstern des Nahen Ostens.

56 Bei dem genannten Kloster handelt es sich um die russisch-orthodoxe Skete des Hl. Chariton, die 1865 von russ. Mönchen wiederentdeckt worden war und Anfang des 20. Jh.s von einer Delegation russ. Mönche vom Athoskloster Panteleemon erworben und wiederbelebt wurde. 
Am 24. Februar verließ ich Jerusalem, um zusammen mit einem praktischen Arzt aus Dresden zu Wagen nach Norden zu fahren. Über die Tour von Jerusalem bis Djennin ist wenig zu sagen. Es war eine genußreiche Fahrt durch den blühenden Frühling. Genauer besichtigte ich in Sebastije die Ausgrabungen der Amerikaner, die auf der höchsten Spitze des Berges einen Tempel mit grandioser Freitreppe ausgegraben haben. ${ }^{57}$ Daneben ein Augustusbild auf breitem Piedestal (das Bild selbst soll nach Konstantinopel gebracht sein). ${ }^{58}$

Von Djennin fuhren wir mit Eisenbahn und Dampfboot nach 'ain Tab$\mathrm{ja}^{59}$ am See Genezareth, wo wir in dem wundervoll gelegenen Hospiz des katholischen Palästinavereins freundliche und gute Aufnahme fanden. Während des mehrtägigen Aufenthalts besuchte ich die Ruinenstätte von Kapernaum, den Franziskanern gehörig, die die große Synagoge fast völlig ausgegraben haben, die Stätte von Bethsaida Julias und Medschdel.

Am 28. Februar ritt ich von Tiberias nach dem Tabor, dessen französisches Kloster nie ausgestorben war $^{60}$, und von dort am Abend nach $\mathrm{Na}$ zareth. Am nächsten Morgen fuhr ich nach Haiffa, besuchte den Karmel und die deutsche Templerkolonie und schiffte mich am Abend an Bord der Tewfikich ${ }^{61}$ ein, um die Heimreise anzutreten. Sie führte mich über Port Said und Alexandrien nach Neapel zurück, wo ich am 7. März ankam.

Auch auf der Rückreise konnte ich es mir nicht versagen, einige wenige [18] Tage auf die Kunstschätze Italiens zu wenden. Am 8.-10. blieb ich in Florenz, am 11. und 12. in Mailand. Am 13. reiste ich über den St. Gotthard und Basel zurück[.]

Nur mit tiefem Dank und hoher Befriedigung kann ich, diesen Bericht schließend, auf die Reise zurücksehen. Was ich erlebt und gelernt kommt freilich in dem Bericht nicht $<$ so $>$ zum Ausdruck, wie ich es gewünscht hätte. Trotzdem hoffe ich, geht auch aus ihm hervor, daß ich die Mittel zu dieser Reise nicht vergeblich erhalten habe. Wenn mein Bericht sich gegen Ende mit einer summarischen Aufzählung begnügt, so bitte ich das

57 Das arabische Dorf Sebastije liegt an der Stelle des antiken Samarias, das Herodes d. Gr. ausbauen und Kaiser Augustus zu Ehren in Sebaste umbenennen ließ. Im Rahmen der sog. Harvard Expedition unter Leitung von Gottlieb Schumacher (1857-1925) und George Andrew Reisner (1867-1942) fanden zwischen 1908 und 1910 erste Ausgrabungen statt, die beträchtliche Teile des antiken Samarias zu Tage brachten; vgl. G. A. ReISNER, Harvard Excavations at Samaria 1908-1910, Vol. I: Text, Cambridge 1924; G. A. Reisner, Harvard Excavations at Samaria 1908-1910, Vol. II: Plans and Plates, Cambridge 1924.

58 Gemeint ist die von Reisner, Harvard Excavations at Samaria 1908-1910, Vol. I: Text, 176 beschriebene ursprünglich ca. $3 \mathrm{~m}$ hohe marmorne Augustusstatue, vgl. die Abb. in ReIsNER, Harvard Excavations at Samaria 1908-1910, Vol. II: Plans and Plates, Plate 79ef.

59 Gemeint ist 'Ain Tâbira.

60 Gemeint ist das 1631 gegründete Franziskaner-Kloster, wobei sich bereits um 1100 Benediktiner auf dem Tabor niedergelassen hatten.

61 Die Tewfikich war ein nach Mohammed Tewfik Pasha (1852-1892), 1879-1892 Vizekönig von Ägypten und Sudan, benannter Dampfer, der im Mittelmeerraum verkehrte. 
damit zu entschuldigen, daß ich jeden Tag meine Einberufung zur Armee erwarte. Ich wollte aber auf alle Fälle den Bericht vorher einreichen.

\section{Die Beilage zum Reisebericht Martin Flashars}

Die 32-seitige „Beilage“ zum Reisebericht Martin Flashars ist mit blauem Buntstift auf der jeweiligen Recto-Seite von 62 bis 77 durchnummeriert. Das Papier hat das Format 33,0 × 21,0 cm. Die Deckseite trägt den Eingangsstempel „Universität Berlin, Eing. 13 Aug. 1914“ samt Aktenvermerk ,zu 2243 S.B. 1/14“" Wie schon beim Reisebericht selbst, so findet sich auch hier die Notiz Alfred Rahlfs: „13. Okt. $1915 \mathrm{zu}$ dauernder Aufbewahrung beim Septuaginta-Unternehmen erhalten. A. Rahlfs." Die erste Seite benennt den Inhalt:

1. Sir. Prolog Z. I,2-III, 2 nach fol. 56 und 27 der Handschrift $\alpha \gamma^{\prime} \tau \alpha \varphi^{\prime} 2$.

2. Fragmente aus Hiob, fol. 205, 4, 164, 167, 3, 206, 195, 200 der Handschrift $\alpha \gamma^{\prime} \sigma \tau \alpha v \varrho^{\prime}$ Nr. 36.

Sowohl die Sirach- als auch die Hiobfragmente sind auf der Grundlage jenes Schriftstïcks von Alfred Rahlfs in angemessener Weise herausgegeben worden, so dass an dieser Stelle lediglich auf die Rahlfs'sche Publikation verwiesen werden kann. ${ }^{62}$

\section{Das Kollationsheftchen zu Ra 929}

Das Kollationsheftchen zu Ra 929 im Format 20,6 × 16,5 cm und im Umfang von 20 Seiten, wobei die letzten drei Seiten unbeschrieben sind, trägt eine handschriftliche Vorbemerkung von der Hand Alfred Rahlfs': „Zugrunde gelegt ist die mangelhafte Ausgabe von J. Rendel Harris, Biblical fragments from Mount Sinai (London 1890), S. 11-14. Aus dieser haben wir die Stellen notiert, an welchen sich erhebliche Varianten finden, und Martin Flashar hat die in der Hs. stehenden Lesarten unterstrichen. Außerdem hat er andere Korrekturen zu Harris gegeben und die von Harris nur zum kleinen Teil herausgegebene Rückseite des ersten Blattes ganz abgeschrieben. (Einige Nachträge habe ich nach einer vollständigen Abschrift Flashars hinzugefügt. A. Rahlfs.) “.

62 Rahlfs, Palimpsest-Fragmente. 
Ho. 56. verto. Cor 1.
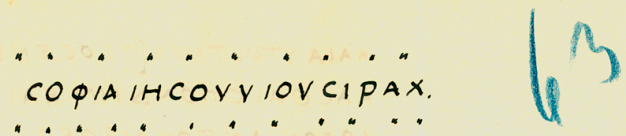

$\Delta N \Delta O K \dot{\Omega}[\dot{M} E \dot{N} \dot{T} \Omega \dot{N} K \dot{K} A \dot{T} \dot{A}$

$\boldsymbol{Z}$ is

THNEPMHNEIAN IIEQIAO

IONHMEN $\Omega N A E \equiv E \Omega N$

A $\triangle$ YNAMEINOY TAP ICOAY

$i_{3}$

S NAMEIAYTA EAYTOICE

BPAICTI NETOMENA KAI

OTAN METAXOHEIEETEPA

14

TA $\Omega C C A N O Y M O N O N \triangle E$

TAYTA AMAA KAI AYTOCO

10 NOMOCKAI OI IPOQHTAI

is

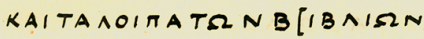

OYMIKPAN EXEITHN

QOPANENEAYTOIC $A$ E [0"

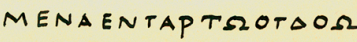

is KAITPIAKOCT \& ETEIE MITOYEYEPTETOY BACI

AESC IAPATENHOEIC

EIC AITY TTON KAICVIXPO

NICACEYPON OVMIKPAC

20 IAIDEIAC AQOMOION

ANATKAIOTATON EOEMHN

i6

if

is

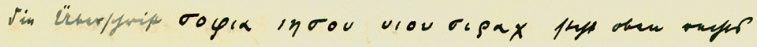

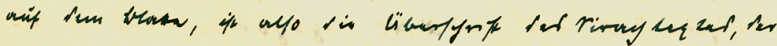

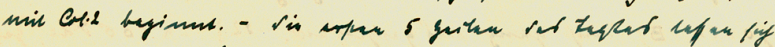

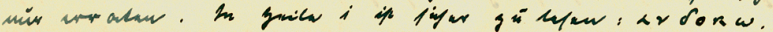

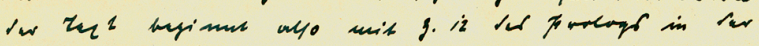

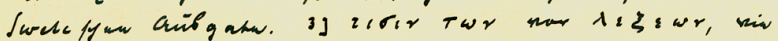

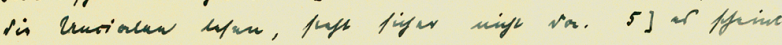

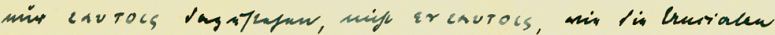

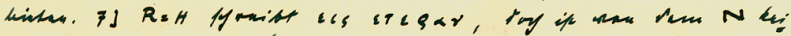

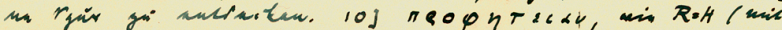

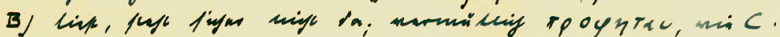

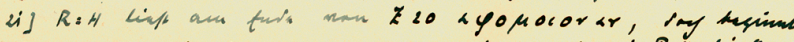

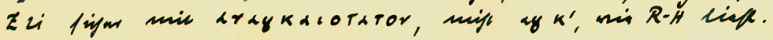

Abb. 2: Die Beilage zum Reisebericht Martin Flashars, f. 63r. 


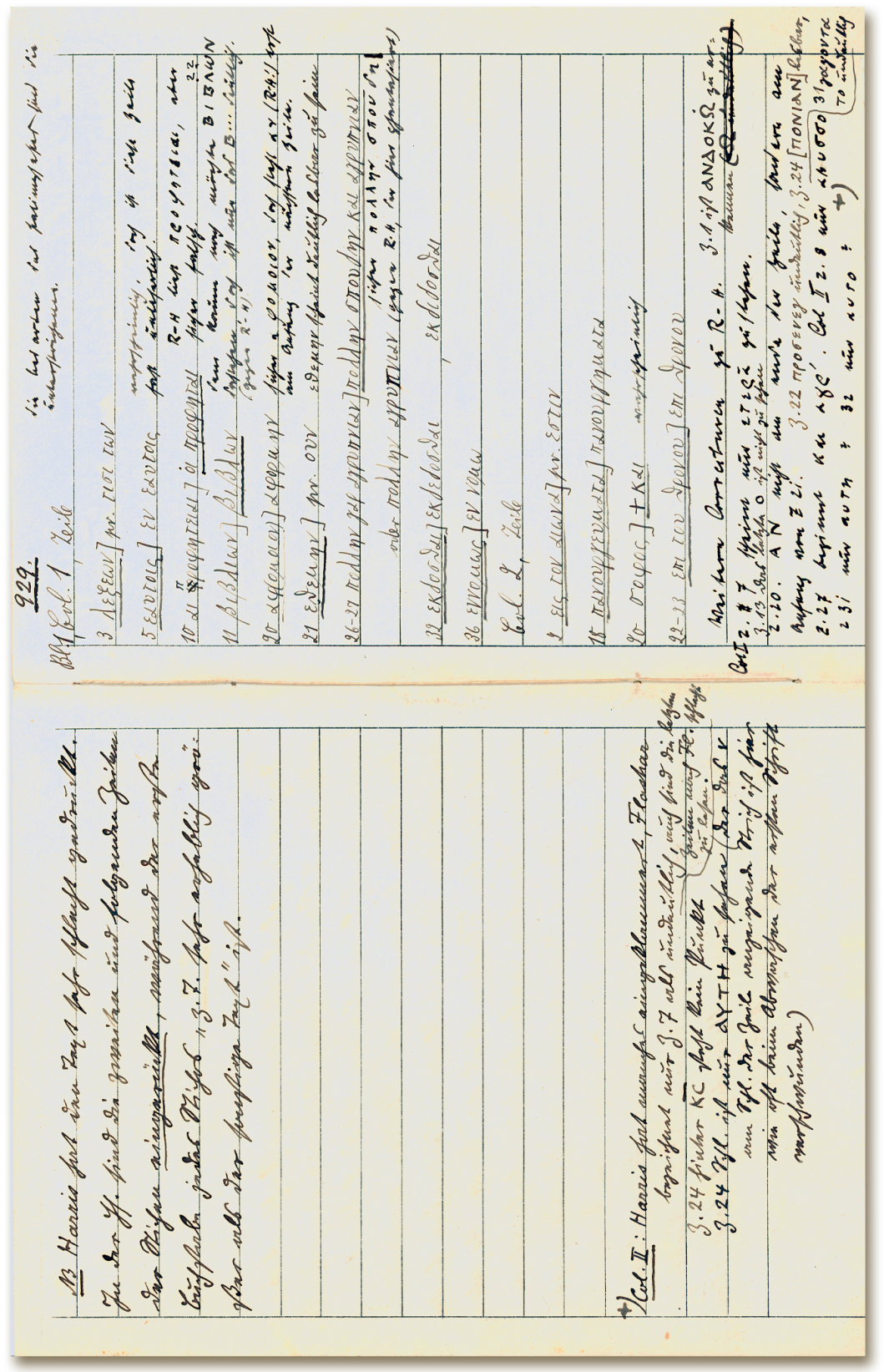

Abb. 3: Das Kollationsheftchen zu Ra 929. 


\title{
6. Eine Postkarte Flashars an Rahlfs vom Juli 1914
}

Postkarte im Format 13,9 × 8,9 cm mit folgendem Poststempel: „BetzinCarwesee, 16.7.1914“. ${ }^{63}$

\author{
Herrn Prof. Rahlfs \\ Hochwürden \\ Göttingen \\ Düstere Eichenweg $19^{64}$ \\ Hochverehrter Herr Professor!
}

Besten Dank für Manuskript und Brief zum Sirachtext: fol 56 verso I32 steht in der Tat $\varkappa \alpha \iota \varepsilon \iota \delta \varepsilon v \delta \varepsilon$ nicht $\varkappa \alpha \iota \varepsilon \iota \sigma \varepsilon \iota \delta \varepsilon v$ wie ich unbegreiflicherweise stehen gelassen habe. Ob dagegen 427 verso 19.20 zu lesen ist $\gamma \varepsilon \varrho \mid \varrho \eta \sigma \alpha \sigma \iota v$ ist mir zweifelhaft. Jedenfalls ist R-H' Lesart hier richtig, sonst hätte ich es vermerkt. Hinsichtlich der letzten Zeile schwanke ich zwischen $\mu \tau \varrho$ und $\mu \eta \varrho$, das letztere war mir, wie meine Notizen zeigen wahrscheinlicher, doch war mir diese Abkürzung unbekannt. Job f. 4. verso 12: ob $\varepsilon \pi \varepsilon \sigma \eta \mu \eta \nu \omega$ kann ich nicht sagen. In meinem Originalmanuskript steht: nur Spuren. f.

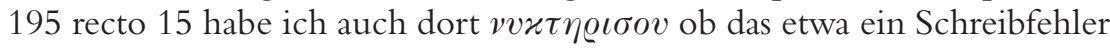
meinerseits ist kann ich nicht sagen. || f. 195 verso ist die Zeilenzählung überhaupt sehr fragwürdig, weil Anfang und Ende unleserlich sind. Tat-

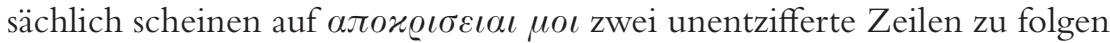
(dann hatte dies Blatt ausnahmsweise 16 Zeilen.) - Der Bericht, als dessen Beilagen die Sept.-sachen gedacht sind, geht an den Senat. An eine Veröffentlichung habe ich nicht gedacht.

Mit bester Empfehlung bin ich Ihr sehr erg. M. F.

Wie aus dem letzten Satz des Schreibens hervorgeht, scheint Rahlfs die Anfrage gehabt zu haben, ob Flashar eine Veröffentlichung seines Reiseberichtes beabsichtigte. Zumindest wird daraus ersichtlich, dass schon Rahlfs den Bericht Flashars anscheinend für publikationswürdig erachtete. Meines Erachtens ist jedoch kaum davon auszugehen, dass Flashar einer Veröffentlichung gegenüber prinzipiell abgeneigt war oder gewesen wäre; immerhin handelt es sich nicht um einen privaten Bericht, sondern um einen von Vornherein für ein, wenn auch kleines,

63 Betzin und Carwesee (heutige Schreibung: „Karwesee“) liegen in Brandenburg, ca. 30 $\mathrm{km}$ nordwestlich von Berlin.

64 Ab dem 20. April 1912 bis Ende Dezember 1919 befand sich das SeptuagintaUnternehmen im Düstere Eichenweg 19; vgl. hierzu Kommission des SePtuagintaUnternehmens, Fünfter Bericht (Berichtsjahr 1912), 23 und Kommission des SeptuaGinTA-Unternehmens, Zwölfter Bericht über das Septuaginta-Unternehmen (Berichtsjahr 1919), in: NGWG. Geschäftliche Mitteilungen 1920, Berlin 1920, 39. 
Publikum, namentlich den Senat der Berliner Friedrich-Wilhelms-Universität bestimmtes Dokument. Was nun Rahlfs damals bereits erkannt haben dürfte, das steht heute auf jeden Fall fest: Die Beobachtungen Flashars bereichern unbestritten unsere Kenntnis von der Situation und den Gegebenheiten in Palästina am Vorabend des 1. Weltkriegs, und dies speziell aus der Sicht eines deutschen Reisenden.

Der Vollständigkeit halber soll im Folgenden noch ein kleiner Überblick über die Handschriftenreisen zwischen den Weltkriegen folgen, die den Handschriftenbestand des Unternehmens weiter ergänzten.

\section{Die Handschriftenreisen zwischen den Weltkriegen}

Der 1. Weltkrieg und vor allem die nachfolgende Krise stellten für die Handschriftenerwerbungen des Septuaginta-Unternehmens einen großen Einschnitt dar. Erst Ende der 20er Jahre konnte die nächste Reise des Unternehmens stattfinden. Der entsprechende Jahresbericht des Unternehmens führt dazu aus: „Herr Studienrat Möhle, unterstützt von stud. Paul Rahlfs, führte von August bis November 1929 eine Reise nach dem Athos, Athen, Patmos und Konstantinopel aus, um die dortigen Septuaginta-Handschriften zu photographieren. Trotz vieler Schwierigkeiten gelang es ihm, in der Zeit von etwa 21/2 Monaten mehr als zehntausend Aufnahmen im Format 2,4 x 3,6 cm zu machen und fertig entwickelt nach Göttingen heimzubringen, wo sie von einem Berufsphotographen auf 18 x $24 \mathrm{~cm}$ vergrößert werden und vollständig lesbare Kopien ergeben. Dadurch ist unser Bestand von Handschriften-Photographien um ein wichtiges und sonst kaum erreichbares Material bereichert" ${ }^{.65}$

65 Kommission des Septuaginta-Unternehmens, Zweiundzwanzigster Bericht über das Septuaginta-Unternehmen (Berichtsjahr 1929), in: NGWG. Geschäftliche Mitteilungen 1929/30, Berlin 1930, 25-26, hier 26. Der Bericht fährt fort: „Als Nebengewinn ergab sich noch, daß Herr Möhle in Konstantinopel den Kommentar Theodorets zu Jesaia, von welchem bisher nur Katenen-Fragmente bekannt waren, aus der einzigen erhaltenen Handschrift photographieren konnte“. Der Bericht des folgenden Jahres fügt ergänzend hinzu: „Demnächst wird er [sc. Hr. Möhle] noch einmal nach Konstantinopel fahren und dort versuchen, aus der vielfach schlecht erhaltenen Handschrift des Theodoret-Kommentars noch manches zu gewinnen, was auf den im Herbst 1929 mitgebrachten Photogrammen nicht sicher zu lesen ist"; Kommission des SePtuaginta-UnterneHmens, Dreiundzwanzigster Bericht über das Septuaginta-Unternehmen (Berichtsjahr 1930), in: NGWG. Geschäftliche Mitteilungen 1930/31, Berlin 1931, 33-35, hier 35. Die von Rahlfs angekündigte zweite Reise Möhles nach Konstantinopel erfolgte 1931, s. u. m. Anm. 74 . 


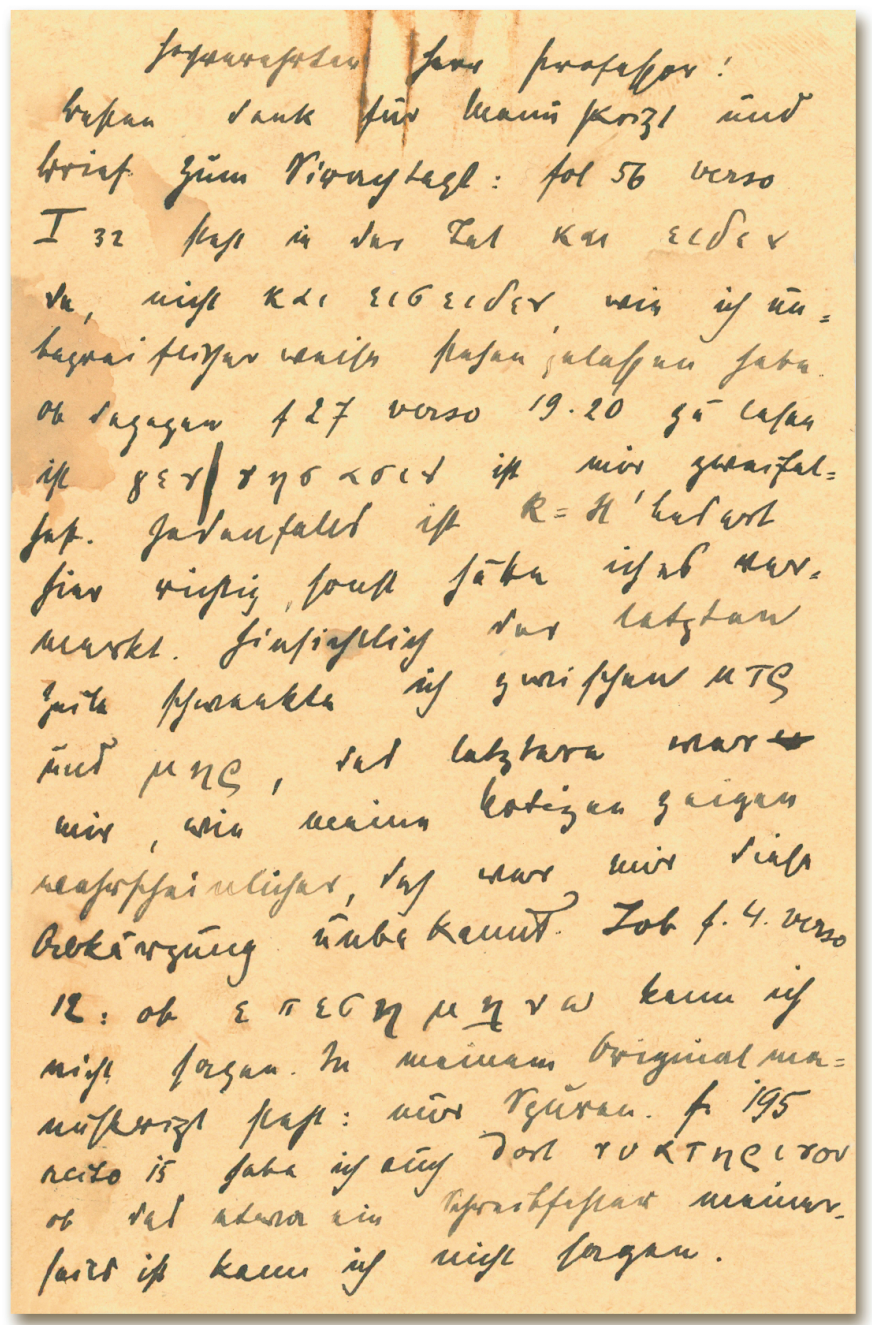

Abb. 4: Die Rückseite der Postkarte Flashars an Rahlfs vom Juli 1914. 
Derselbe Studienrat Möhle führte im Folgejahr eine Italienreise durch. Im Bericht heißt es dazu: Er ,ist zu diesem Zwecke von April bis Juni 1930 in Italien gewesen, wobei er zugleich viele andere Handschriften italienischer Bibliotheken zu den verschiedensten alttestamentlichen Büchern photographiert hat " ${ }^{66}$ Auch im folgenden Jahr war Möhle für das Septuaginta-Unternehmen auf Forschungsreise: „Herr Studienrat Möhle besuchte im April und Mai 1931 Konstantinopel und Patmos, um sein Material für die Ausgabe des neu entdeckten Kommentars Theodorets zu Jesaia zu kontrollieren und zu vervollständigen “ ${ }^{67}$ Über den Handschriftenbestand des Unternehmens heißt es im Bericht für das Jahr 1932: „Eine Schwester des Herrn Deicke, die Bibliothekarin Frl. Hanna Deicke, hat die Handbibliothek des Septuaginta-Unternehmens (etwa 500 Bände) und die Sammlung von Handschriften-Photographien (etwa 50000 Blätter), deren viele, erst in den letzten Jahren hinzugekommen, noch der Ordnung bedurften, vollständig geordnet und katalogisiert, sodaß jetzt alles bequem zu benutzen ist" ${ }^{6}{ }^{68}$

Die Handschriftenreisen zwischen den Weltkriegen im Überblick

\begin{tabular}{|c|c|c|}
\hline $\begin{array}{l}\text { Griechenland, } \\
\text { Kleinasien } \\
\text { (Athos, } \\
\text { Athen, Patmos, } \\
\text { Konstantinopel) }\end{array}$ & $1929^{69}$ & $\begin{array}{l}\text { August Möhle }{ }^{70} \text {, Paul Rahlfs } \\
\text { Photographien (10.000 Aufnahmen): }:^{72} \text { Athen (Ra 313-315; 342); } \\
\text { Athos Vatopedi (Ra 317-321); Athos Iviron (Ra 329-333; 336- } \\
\text { 338); Athos Koutloumousiou (Ra 339-341); Patmos (Ra 612-615; } \\
\text { 617-620); Konstantinopel (Ra 397-401) }\end{array}$ \\
\hline
\end{tabular}

66 Kommission des Septuaginta-Unternehmens, Dreiundzwanzigster Bericht (Berichtsjahr 1930), 35.

67 Kommission des Septuaginta-Unternehmens, 24. Bericht über das Septuaginta-Unternehmen (Berichtsjahr 1931), in: NGWG. Geschäftliche Mitteilungen 1931/32, Berlin 1932, 29.

68 Kommission des Septuaginta-Unternehmens, 25. Bericht über das Septuaginta-Unternehmen, in: NGWG. Geschäftliche Mitteilungen 1932/33, Berlin 1933, 32-33, hier 33.

69 Den Angaben Möhles zufolge erfolgte die Reise näherhin in folgendem Zeitraum: Ende August bis Ende Oktober 1929 verbrachten August Möhle und Paul Rahlfs in Athen, auf dem Athos und auf Patmos; Anfang November besuchte Möhle die Bibliothek des Metochions in Konstantinopel; die Photographien der berühmten Theodoret-Hs. fertigte er am 2. November an. Vgl. insgesamt A. MöHLE, Theodoret von Kyros, Kommentar zu Jesaia (MSU 5), Berlin 1932, VI.

70 Dr. August Möhle (7.3.1885-29.9.1971), Theologe und Philologe, war Gymnasiallehrer sowie 1926-1937 Mitarbeiter des Septuaginta-Unternehmens. 1932 promovierte er über den Jesaja-Kommentar des Theodoret von Kyros. Während des 2. Weltkriegs arbeitete Möhle als Hauslehrer in Florenz. 1950 ging er in Pension.

71 Alfred Rahlfs' Sohn Paul (26.10.1909-9.10.1987) arbeitete mehrere Semester als stud. Hilfskraft im Septuaginta-Unternehmen.

72 Die Photographien von 1929 haben ein Format von $18 \times 24$ cm; die Gießener Kapseln haben eine Höhe von 21 cm; s.o. m. Anm. 23. 
Die Handschriftenreisen zwischen den Weltkriegen im Überblick (Fortsetzung)

\begin{tabular}{|c|c|c|}
\hline Italien & 1930 & $\begin{array}{l}\text { August Möhle } \\
\text { Photographien: } \\
\text { Grottaferrata (392); Mailand (443-446; 449-452); Messina (455- } \\
\text { 460) }\end{array}$ \\
\hline $\begin{array}{l}\text { Kleinasien } \\
\text { (Konstantinopel, } \\
\text { Patmos) }\end{array}$ & $1931^{74}$ & $\begin{array}{l}\text { August Möhle } \\
\text { Handschriftenkonsultation }\end{array}$ \\
\hline
\end{tabular}

\section{Literatur}

Albrecht, F., Art. „Duensing, Hugo Berthold Heinrich“, in: BBKL 33, Nordhausen 2012, 307-318.

- Art. „Flashar, Martin Johannes Gerhard“, in: BBKL 33, Nordhausen 2012, 418-420.

- Between Boon and Bane. The Use of Chemical Reagents in Palimpsest Research in the Nineteenth Century, in: Care and Conservation of Manuscripts 13, Kopenhagen 2012, 147-165.

Bernet, C., Art. „Harris, James Rendel“, in: BBKL 30, Nordhausen 2009, 557-569.

Carmel, A., Die Siedlungen der württembergischen Templer in Palästina 1868-1918. Ihre lokalpolitischen und internationalen Probleme. Aus dem Hebräischen übersetzt von P. Leshem (Veröffentlichungen der Kommission für geschichtliche Landeskunde in Baden-Württemberg. Reihe B, Forschungen 77), Stuttgart ${ }^{3} 2000$.

Dalman, G., Arbeit und Sitte in Palästina, Vol. 1: Jahreslauf und Tageslauf. 2. Hälfte, Frühling und Sommer (SDPI 3/2, zugleich BFChTh, II/15), Gütersloh 1928.

- Arbeit und Sitte in Palästina, Vol. 2: Der Ackerbau (SDPI 5, zugleich BFChTh, II/27), Gütersloh 1932.

- Arbeit und Sitte in Palästina, Vol. 5: Webstoff, Spinnen, Weben, Kleidung (SDPI 8, zugleich BFChTh, II/36), Gütersloh 1937.

Dölger, F., Art. „Dieterich, Karl Gustav“, in: NDB 3, Berlin 1957, 671-672.

EISLER, J., Das Johanniterhospiz in Jerusalem, Berlin 2008.

- Die Auguste-Victoria-Stiftung auf dem Ölberg in Jerusalem. Eine Bilddokumentation (Kleine Schriften des Vereins für württembergische Kirchengeschichte 8), Stuttgart 2010.

Eliav, M. (ed.), Die Juden Palästinas in der deutschen Politik. Dokumente aus dem Archiv des deutschen Konsulats in Jerusalem, 1842-1914, Tel Aviv 1973.

Harris, J. R., Biblical Fragments from Mount Sinai, London 1890.

Irmscher, J., Karl Dieterich und die Begründung der Neogräzistik in Leipzig, in: J. WerNER (ed.), Modernes Griechenland, modernes Zypern. Vorträge einer wissenschaftlichen Konferenz des Fachbereichs „Antike Literatur/Neogräzistik“ der Karl-MarxUniversität Leipzig, Amsterdam 1989, 244-261.

73 Die Photographien von 1930 haben ebenfalls ein Format von $18 \times 24 \mathrm{~cm}$ und die Gießener Kapseln eine Höhe von $21 \mathrm{~cm}$.

74 Mai 1931 verbrachte Möhle „einige Wochen lang im Metochion“; vgl. MöHLE, Theodoret von Kyros, Kommentar zu Jesaia, VII. 
Kommission des Septuaginta-Unternehmens, Bericht über das Septuaginta-Unternehmen der Kgl. Gesellschaft der Wissenschaften zu Göttingen, in: NGWG. Geschäftliche Mitteilungen 1909, Berlin 1909, 129-138.

- Dritter Bericht über das Septuaginta-Unternehmen (Berichtsjahr 1910), in: NGWG. Geschäftliche Mitteilungen 1911, Berlin 1911, 21-25.

- Vierter Bericht über das Septuaginta-Unternehmen (Berichtsjahr 1911), in: NGWG. Geschäftliche Mitteilungen 1912, Berlin 1912, 18-20.

- Fünfter Bericht über das Septuaginta-Unternehmen (Berichtsjahr 1912), in: NGWG. Geschäftliche Mitteilungen 1913, Berlin 1913, 23-24.

- Sechster Bericht über das Septuaginta-Unternehmen (Berichtsjahr 1913), in: NGWG. Geschäftliche Mitteilungen 1914, Berlin 1914, 22-24.

- Bericht über das Septuaginta-Unternehmen der Königl. Gesellschaft der Wissenschaften zu Göttingen. Der Internationalen Assoziation der Akademien vorgelegt zur Generalversammlung 1913, Göttingen 1913, 1-8.

- Siebenter Bericht über das Septuaginta-Unternehmen (Berichtsjahr 1914), in: NGWG. Geschäftliche Mitteilungen 1915, Berlin 1915, 19-21.

- Achter Bericht über das Septuaginta-Unternehmen (Berichtsjahr 1915), in: NGWG. Geschäftliche Mitteilungen 1916, Berlin 1916, 15-17.

- Zwölfter Bericht über das Septuaginta-Unternehmen (Berichtsjahr 1919), in: NGWG. Geschäftliche Mitteilungen 1920, Berlin 1920, 39.

- Zweiundzwanzigster Bericht über das Septuaginta-Unternehmen (Berichtsjahr 1929), in: NGWG. Geschäftliche Mitteilungen 1929/30, Berlin 1930, 25-26.

- Dreiundzwanzigster Bericht über das Septuaginta-Unternehmen (Berichtsjahr 1930), in: NGWG. Geschäftliche Mitteilungen 1930/31, Berlin 1931, 33-35.

- 24. Bericht über das Septuaginta-Unternehmen (Berichtsjahr 1931), in: NGWG. Geschäftliche Mitteilungen 1931/32, Berlin 1932, 29.

- 25. Bericht über das Septuaginta-Unternehmen, in: NGWG. Geschäftliche Mitteilungen 1932/33, Berlin 1933, 32-33.

Krämer, G., Geschichte Palästinas. Von der osmanischen Eroberung bis zur Gründung des Staates Israel (Beck'sche Reihe 1461), München ${ }^{2} 2002$.

Kreuzenbeck, J., Art. „Tisserant, Eugène“", in: BBKL 20, Nordhausen 2002, 1463-1466.

Krumbacher, K., Die Photographie im Dienste der Geisteswissenschaften, in: J. Ilberg (ed.), Neue Jahrbücher für das klassische Altertum, Geschichte und deutsche Literatur 9 (NJKA 17), Leipzig 1906, 601-659 (Mit fünfzehn Tafeln).

Männchen, J., Gustaf Dalmans Leben und Wirken in der Brüdergemeinde, für die Judenmission und an der Universität Leipzig 1855-1902 (ADPV 9/1), Wiesbaden 1987.

- Gustaf Dalman als Palästinawissenschaftler in Jerusalem und Greifswald 1902-1941 (ADPV 9/2), Wiesbaden 1991.

Maspero, G., Guide du visiteur au Musée du Caire, Kairo ${ }^{3} 1914$.

MayoreK, Y., Zwischen Ost und West. Edmond de Rothschild und Palästina, in: G. Heuberger (ed.), Die Rothschilds. Vol. 2, Beiträge zur Geschichte einer europäischen Familie, Sigmaringen 1994, 133-150.

MöHle, A., Theodoret von Kyros, Kommentar zu Jesaia (MSU 5), Berlin 1932.

Nothnagle, A. (ed.), Seht, wir gehen hinauf nach Jerusalem. Festschrift zum 150jährigen Jubiläum von Talitha Kumi und des Jerusalemvereins, Leipzig 2001.

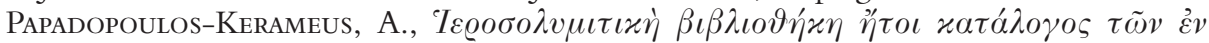

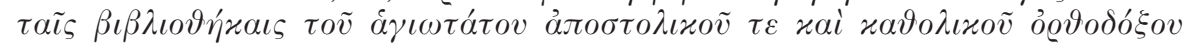

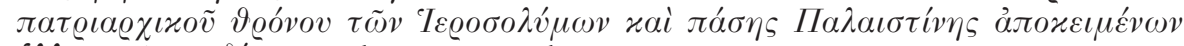

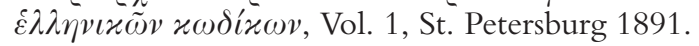


Rahlfs, A., Palimpsest-Fragmente des Sirach und Iob aus Jerusalem. Nach der Entzifferung von Martin Flashar, in: Kleine Mitteilungen aus dem Septuaginta-Unternehmen (MSU 1/7), Berlin 1915, 388-404.

- Verzeichnis der griechischen Handschriften des Alten Testaments. Für das SeptuagintaUnternehmen aufgestellt (MSU 2), Berlin 1914.

Reimpell, W., Geschichte der babylonischen und assyrischen Kleidung, ed. E. Meyer, Berlin 1921.

Reisner, G. A., Harvard Excavations at Samaria 1908-1910, Vol. I: Text, Cambridge 1924.

- Harvard Excavations at Samaria 1908-1910, Vol. II: Plans and Plates, Cambridge 1924.

SAdmon, Z.W., Die Gründung des Technions in Haifa im Lichte deutscher Politik 1907-1920 (EHKB 78), München 1994.

Schmidt, C., Bericht des wissenschaftlichen Beamten Prof. Karl Schmidt über seine Forschungsreise nach dem Katharinenkloster auf dem Sinai, in: SPAW 1915, Berlin 1915, 122-125.

Schmidt, C. / B. Moritz, Die Sinai-Expedition im Frühjahr 1914, in: SPAW.PH 7, Berlin 1926, 26-34.

Schwake, N., Die Entwicklung des Krankenhauswesens der Stadt Jerusalem vom Ende des 18. bis zum Beginn des 20. Jahrhunderts (Studien zur Geschichte des Krankenhauswesens 8), Herzogenrath 1983.

Tisserant, E., Un manuscrit palimpseste de Job, in: RB 21 (1912), 481-503.

Tromm, A., Concordantiae Graecae in Septuaginta Interpretes. 2 Vol., Amstelodami/Trajecti ad Rhenum 1718.

Wawrzyn, H., Ham and Eggs in Palestine. The Auguste Victoria Foundation 1898-1939, Marburg 2005.

Wesseling, K.-G., Art. „Schmidt, Carl“, in: BBKL 9, Herzberg 1995, 443-446.

Wulf, S., Jerusalem, Aleppo, Konstantinopel. Der Hamburger Tropenmediziner Peter Mühlens im Osmanischen Reich am Vorabend und zu Beginn des Ersten Weltkriegs (Hamburger Studien zur Geschichte der Medizin 5), Münster 2005.

Ziegler, J., Sapientia Jesu Filii Sirach (Septuaginta. Vetus Testamentum Graecum Auctoritate Academiae Scientiarum Gottingensis editum XII/2), Göttingen ${ }^{2} 1981$. 
